\title{
Propulsion Theory of Flapping Airfoils, Comparison with Computational Fluid Dynamics
}

\author{
D. F. Hunsaker ${ }^{*}$ and W. F. Phillips ${ }^{\dagger}$ \\ Utah State University, Logan, Utah 84322-4130
}

\begin{abstract}
The thrust, required power, and propulsive efficiency of a flapping airfoil as predicted by the well-known Theodorsen model are compared with solutions obtained from gridresolved inviscid computational fluid dynamics. A straight-forward summary of Theodorsen's flapping airfoil model is presented using updated terminology and symbols. This shows that both axial and normal reduced frequencies are of significant importance. The axial reduced frequency is based on the chord length and the normal reduced frequency is based on the plunging amplitude. Computational fluid dynamics solutions are presented over the range of both reduced frequencies typically encountered in the forward flight of birds. It is shown that computational results agree reasonably well with those predicted by Theodorsen's model at low flapping frequencies. An alternate model is also developed, which shows that the time-dependent aerodynamic forces acting on a flapping airfoil can be related to two unknown Fourier coefficients. The computational results are correlated with algebraic relations for these Fourier coefficients, which can be used to predict the thrust, required power, and propulsive efficiency for airfoils with sinusoidal pitching and plunging motion.
\end{abstract}

\section{Nomenclature}

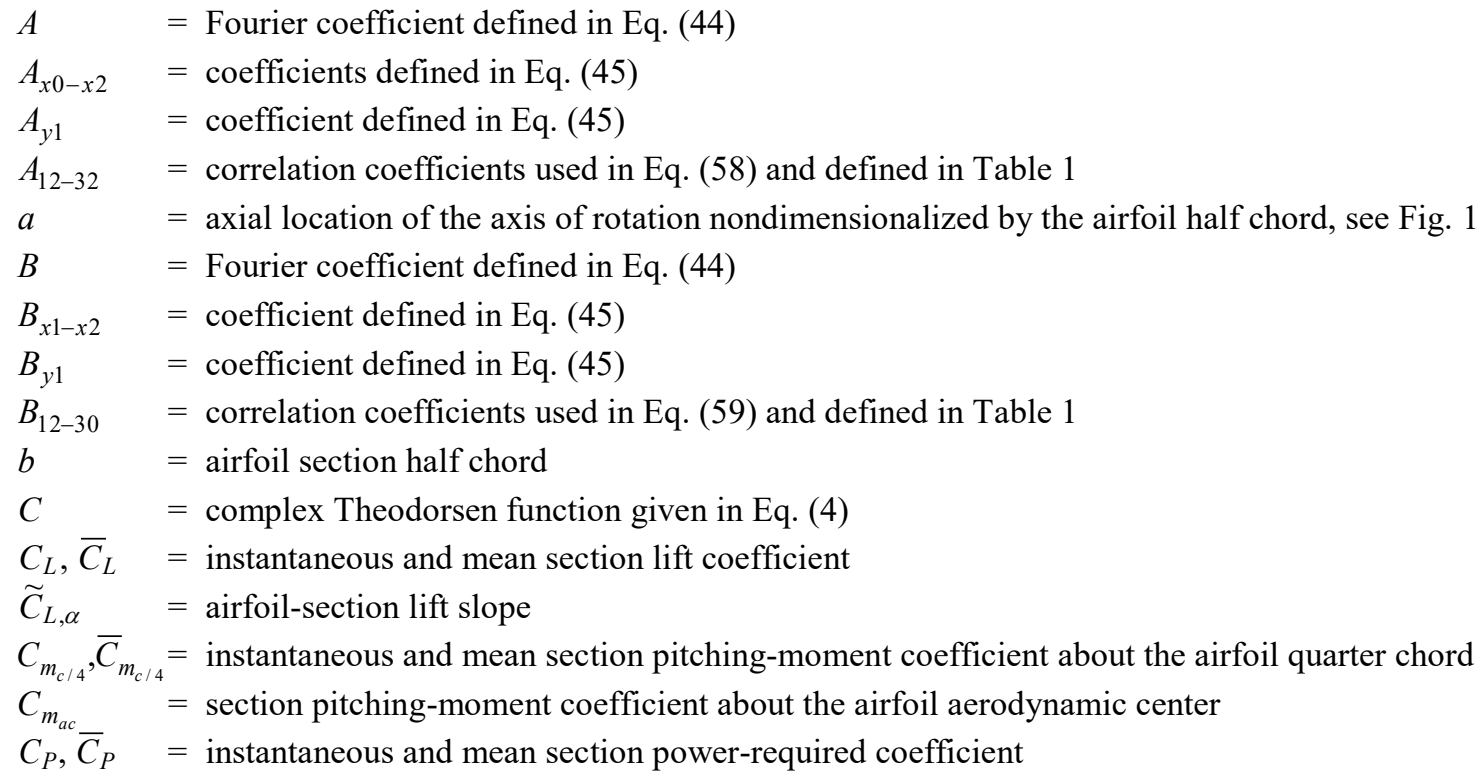

\footnotetext{
* Adjunct Professor, Mechanical and Aerospace Engineering Department, 4130 Old Main Hill. Member AIAA.

${ }^{\dagger}$ Emeritus Professor, Mechanical and Aerospace Engineering Department, 4130 Old Main Hill. Senior Member AIAA.
} 


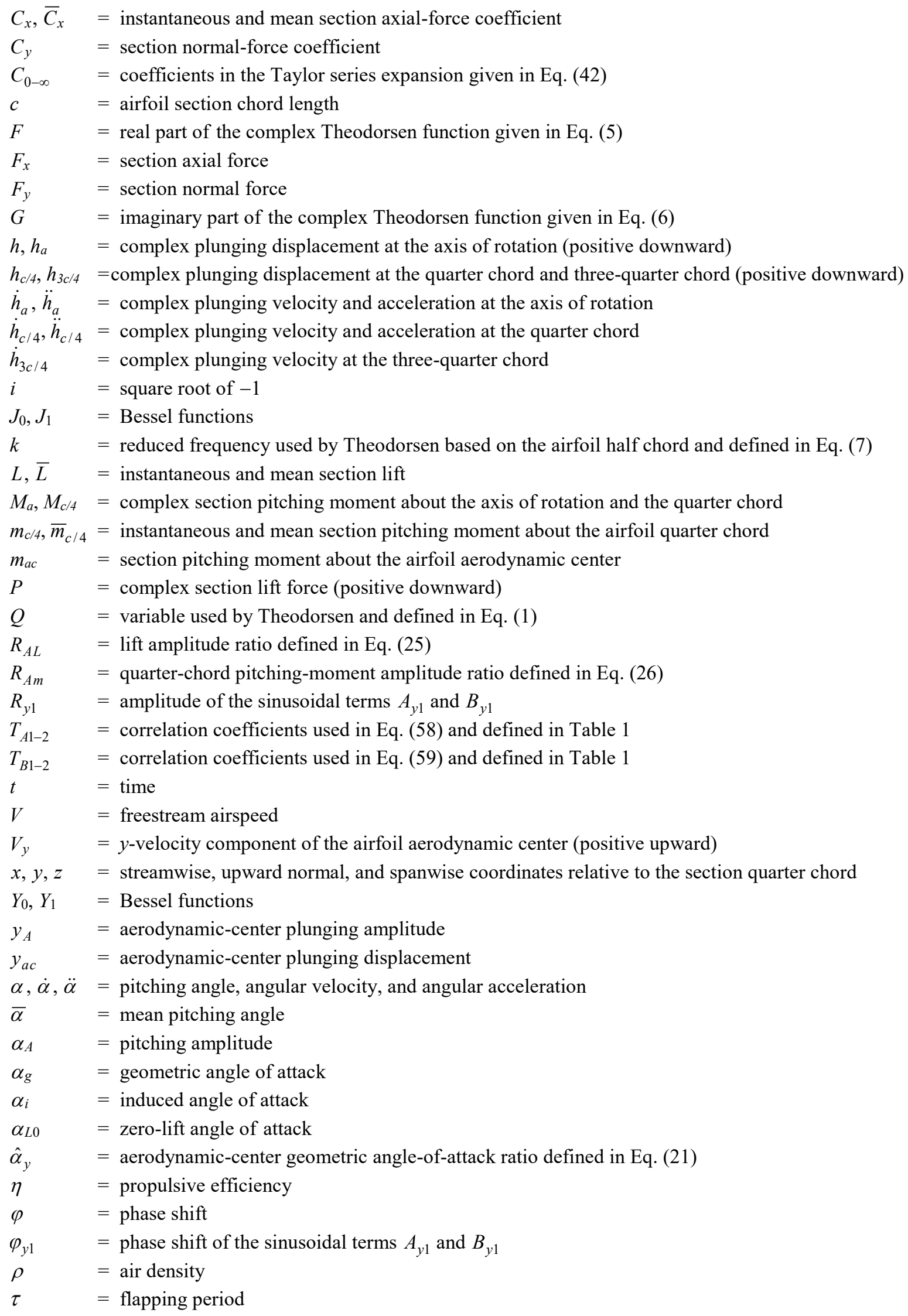




\section{Introduction}

$\mathrm{T}^{\mathrm{H}}$ HE lift from a plunging airfoil is rotated forward through an incremental angle of attack, which, within the small angle approximation, is proportional to the plunging velocity. The rotation of the lift vector through this angle results in the production of thrust. Low-frequency two-dimensional quasi-steady effects of the physics for the production of thrust by a flapping airfoil were presented first by Knoller ${ }^{1}$, and independently by Betz ${ }^{2}$. A decade later, Katzmayr ${ }^{3}$ demonstrated the production of thrust experimentally using the Knoller-Betz low-frequency flapping-wing model.

Aerodynamic forces and moments acting on an airfoil in oscillatory motion are affected by the oscillating vortex wake downstream from the airfoil. The oscillating vorticity in the wake induces fluid motion near the airfoil, which alters the pressure distribution acting on the airfoil relative to that experienced in steady flow. At low frequencies, this effect is small. However, at higher frequencies, the influence of the oscillating wake on the aerodynamics of the airfoil is significant. The foundation for much of the analytical work on oscillating airfoils can be traced back to the work of Wagner ${ }^{4}$, who calculated the vorticity in the wake of an airfoil with non-uniform motion. A few years later, Glauert ${ }^{5}$ was among the first to develop relations for the lift and pitching moment acting on an oscillating airfoil. A review of Glauert's work was presented by von Kármán \& Burgers, ${ }^{6}$ who added an analytical expression for the propulsive force developed by the airfoil.

Theodorsen ${ }^{7}$ developed an analytical model for oscillating airfoils based on conformal mapping, and he presented expressions for the lift and pitching moment acting on an airfoil with three degrees of freedom. However, because his work was focused on understanding the mechanism of flutter, Theodorsen omitted the calculation of the force in the streamwise direction. Later, Garrick ${ }^{8}$ used Theodorsen's mathematical model to extend the work of von Kármán and Burgers ${ }^{6}$ to an airfoil with three degrees of freedom, and he presented expressions for the axial force and propulsive efficiency of a flapping airfoil. Theodorsen's ${ }^{7}$ mathematical model with Garrick's ${ }^{8}$ extension has been widely used to predict the aerodynamics of flapping airfoils.

The approximations underlying Theodorsen's ${ }^{7}$ potential-flow solution include those of a thin airfoil with small camber, small angles of attack, small-amplitude sinusoidal oscillations, and a planar wake with oscillating vorticity. As with the aerodynamic theory of steady flow, analytical results based on these seemingly constraining approximations often give reasonable results well outside the range of parameters for which these approximations might be expected to hold.

Recently, most investigations of wing flapping have focused on experimental studies ${ }^{9-24}$ and numerical solutions that use either potential-flow theory ${ }^{25-37}$ or computational fluid dynamics (CFD). ${ }^{38-54}$ Because the Theodorsen ${ }^{7}$ model appears to be applicable over the range of frequencies and amplitudes that are of interest in practical flapping flight, this model is often used for comparison in CFD and experimental studies, ${ }^{44,47,55,56}$ which show varying degrees of agreement with the Theodorsen model.

For the special case of combined plunging and pitching motion, Theodorsen ${ }^{7}$ considered a thin symmetric airfoil of half chord $b$ represented by the straight line shown in Fig. 1. The airfoil is assumed to undergo a sinusoidal vertical displacement, $h(t)$, at what he refers to as the axis of rotation, which is a dimensionless distance $x / b=a$ aft of the airfoil section half chord. This is combined with a small-angle sinusoidal rotation, $\alpha(t)$, of the entire airfoil. Both $h$ and $\alpha$ were assumed to have zero mean values, $h$ is positive downward, and $\alpha$ is positive clockwise relative to the constant freestream velocity $V$, which approaches the airfoil from the left.

Because the angle $\alpha$ is time dependent, the instantaneous downward displacement of each point on the chord line is different. Only the axis of rotation has the downward displacement $h$, as defined by Theodorsen. ${ }^{7}$ Hence, in the development presented here we shall consistently subscript the symbol $h$ to indicate the point considered. For example, the downward displacement of the axis of rotation will be explicitly denoted here as $h_{a}$. In Theodorsen's ${ }^{7}$ presentation, the downward displacements of other points on the airfoil are of special significance. For example, the small-angle downward displacement of the section quarter-chord point is $h_{c / 4}(t)=h_{a}(t)-(a+1 / 2) b \alpha(t)$, and the small-angle downward displacement of the section three-quarter-chord point is $h_{3 c / 4}(t)=h_{a}(t)-(a-1 / 2) b \alpha(t)$. 


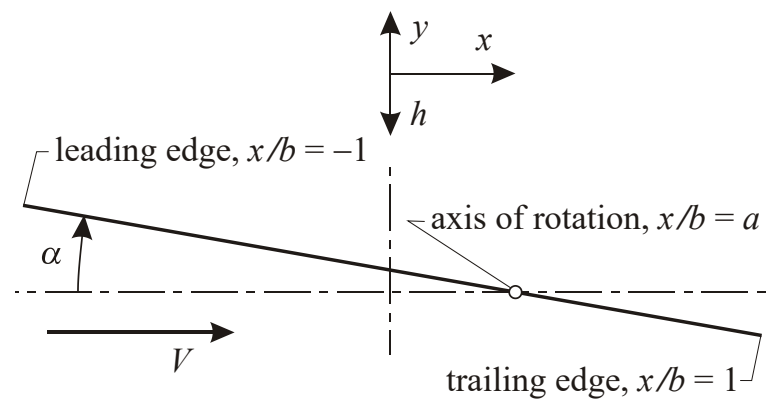

Figure 1. Parameters used by Theodorsen ${ }^{7}$ to describe combined plunging and pitching motion.

Theodorsen ${ }^{7}$ defined the variable $Q(t)$ such that $Q / V$ is physically the instantaneous small-angle geometric angle of attack at the airfoil section three-quarter chord point, i.e.,

$$
\frac{Q}{V} \equiv \frac{\dot{h}_{3 c / 4}}{V}+\alpha=\frac{\dot{h}_{a}}{V}+\left(\frac{1}{2}-a\right) \frac{b \dot{\alpha}}{V}+\alpha
$$

Using potential flow and conformal mapping, Theodorsen ${ }^{7}$ developed an analytical expression for the complex lift (positive downward), which is given by the relation

$$
P(t)=-2 \pi \rho V^{2} b\left[\frac{Q}{V} C(k)+\frac{b \ddot{h_{a}}}{2 V^{2}}-\frac{a b^{2} \ddot{\alpha}}{2 V^{2}}+\frac{b \dot{\alpha}}{2 V}\right]
$$

Similarly, the complex pitching moment about the axis of rotation as presented by Theodorsen ${ }^{7}$ is

$$
M_{a}(t)=2 \pi \rho V^{2} b^{2}\left[\left(a+\frac{1}{2}\right) \frac{Q}{V} C(k)+\frac{a}{2} \frac{b \ddot{h}_{a}}{V^{2}}-\left(\frac{1}{16}+\frac{a^{2}}{2}\right) \frac{b^{2} \ddot{\alpha}}{V^{2}}+\left(\frac{a}{2}-\frac{1}{4}\right) \frac{b \dot{\alpha}}{V}\right]
$$

where

$$
\begin{gathered}
C(k)=F(k)+i G(k) \\
F(k)=\frac{J_{1}(k)\left[J_{1}(k)+Y_{0}(k)\right]+Y_{1}(k)\left[Y_{1}(k)-J_{0}(k)\right]}{\left[J_{1}(k)+Y_{0}(k)\right]^{2}+\left[Y_{1}(k)-J_{0}(k)\right]^{2}} \\
G(k)=-\frac{Y_{1}(k) Y_{0}(k)+J_{1}(k) J_{0}(k)}{\left[J_{1}(k)+Y_{0}(k)\right]^{2}+\left[Y_{1}(k)-J_{0}(k)\right]^{2}}
\end{gathered}
$$

Theodorsen ${ }^{7}$ defined the reduced frequency, $k$, based on the airfoil section half chord, $b$, i.e.,

$$
k \equiv \omega b / V
$$

The functions $F(k)$ and $G(k)$ are of fundamental importance in Theodorsen's ${ }^{7}$ theory of oscillating airfoils. These functions are shown graphically in Fig. 2 plotted as a function of $1 / k$, exactly as they were first presented by Theodorsen ${ }^{7}$ in 1935. Note that $F$ approaches 1.0 in the low-frequency limit and 0.5 in the high-frequency limit, whereas $G$ approaches 0.0 in both limits.

Today it is more conventional to describe airfoil rotations and pitching moments about the section aerodynamic center. Because Theodorsen assumed an infinitely thin airfoil, the aerodynamic center for his model is located at the section quarter chord. Using the relations

$$
\dot{h}_{c / 4}=\dot{h}_{a}-(a+1 / 2) b \dot{\alpha}
$$




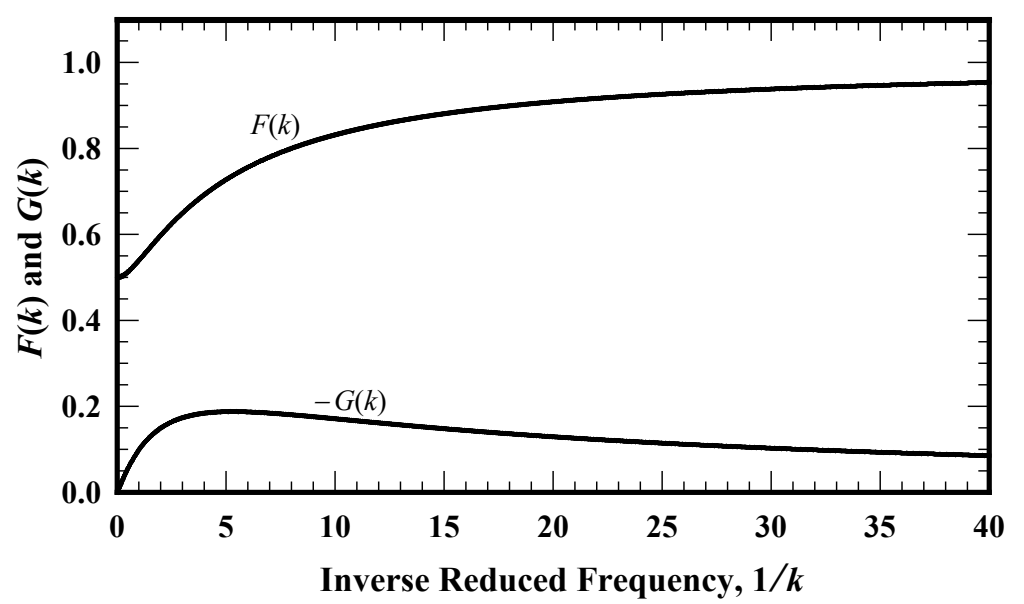

Figure 2. The functions $F(k)$ and $G(k)$ as first presented by Theodorsen ${ }^{7}$ in 1935.

$$
M_{c / 4}=M_{a}+(a+1 / 2) b P
$$

the solution given in Eqs. (1)-(3) is easily transformed to that based on the quarter-chord reference point, i.e.,

$$
\begin{gathered}
\frac{Q}{V} \equiv \frac{\dot{h}_{c / 4}}{V}+\frac{b \dot{\alpha}}{V}+\alpha \\
P(t)=-2 \pi \rho V^{2} b\left[\frac{Q}{V} C(k)+\frac{b \ddot{h}_{c / 4}}{2 V^{2}}+\frac{b^{2} \ddot{\alpha}}{4 V^{2}}+\frac{b \dot{\alpha}}{2 V}\right] \\
M_{c / 4}(t)=-2 \pi \rho V^{2} b^{2}\left(\frac{b \ddot{h}_{c / 4}}{4 V^{2}}+\frac{3 b^{2} \ddot{\alpha}}{16 V^{2}}+\frac{b \dot{\alpha}}{2 V}\right)
\end{gathered}
$$

In Theodorsen's ${ }^{7}$ presentation, he allowed for a phase shift between the plunging and pitching. Theodorsen ${ }^{7}$ defined this phase shift as the angle between the pitching rotation and the downward plunging displacement at his chosen reference point, $x / b=a$. Because we are using the airfoil quarter chord as our reference point, for greatest simplicity, it will be more convenient to define this phase shift as the angle between the pitching rotation, $\alpha(t)$, and the quarter-chord $y$-velocity component (positive upward), $V_{y}(t)=-\dot{h}_{c / 4}(t)$. Hence, the position, velocity, and acceleration of the quarter-chord plunging motion as well as the airfoil pitching motion can be described in complex form as

$$
\begin{gathered}
h_{c / 4}(t)=-y_{A} e^{i \omega t}=\left[-y_{A} \cos (\omega t)\right]+i\left[-y_{A} \sin (\omega t)\right] \\
\dot{h}_{c / 4}(t)=-i \omega y_{A} e^{i \omega t}=\left[\omega y_{A} \sin (\omega t)\right]+i\left[-\omega y_{A} \cos (\omega t)\right] \\
\ddot{h}_{c / 4}(t)=\omega^{2} y_{A} e^{i \omega t}=\left[\omega^{2} y_{A} \cos (\omega t)\right]+i\left[\omega^{2} y_{A} \sin (\omega t)\right] \\
\alpha(t)=i \alpha_{A} e^{i(\omega t+\varphi)}=\left[-\alpha_{A} \sin (\omega t+\varphi)\right]+i\left[\alpha_{A} \cos (\omega t+\varphi)\right] \\
\dot{\alpha}(t)=-\omega \alpha_{A} e^{i(\omega t+\varphi)}=\left[-\omega \alpha_{A} \cos (\omega t+\varphi)\right]+i\left[-\omega \alpha_{A} \sin (\omega t+\varphi)\right] \\
\ddot{\alpha}(t)=-i \omega^{2} \alpha_{A} e^{i(\omega t+\varphi)}=\left[\omega^{2} \alpha_{A} \sin (\omega t+\varphi)\right]+i\left[-\omega^{2} \alpha_{A} \cos (\omega t+\varphi)\right]
\end{gathered}
$$


Adding the effects of mean lift and finite thickness, Theodorsen's ${ }^{7}$ solution for the traditional section lift coefficient can be obtained from Eqs. (4), (10), and (11), i.e.,

$$
C_{L}(t) \equiv \frac{\bar{L}-\operatorname{imag}[P(t)]}{\frac{1}{2} \rho V^{2}(2 b)}=\bar{C}_{L}+\widetilde{C}_{L, \alpha} \operatorname{imag}\left[\left(\frac{\dot{h}_{c / 4}}{V}+\frac{b \dot{\alpha}}{V}+\alpha\right)(F+i G)+\frac{b \ddot{h}_{c / 4}}{2 V^{2}}+\frac{b^{2} \ddot{\alpha}}{4 V^{2}}+\frac{b \dot{\alpha}}{2 V}\right]
$$

or after applying Eqs. (13)-(18),

$$
\begin{gathered}
C_{L}(t)=\bar{C}_{L}-\widetilde{C}_{L, \alpha} \hat{\omega}_{y}\left[\cos (\omega t)+k \hat{\alpha}_{y} \sin (\omega t+\varphi)-\hat{\alpha}_{y} \cos (\omega t+\varphi)\right] F \\
+\widetilde{C}_{L, \alpha} \hat{\omega}_{y}\left[\sin (\omega t)-k \hat{\alpha}_{y} \cos (\omega t+\varphi)-\hat{\alpha}_{y} \sin (\omega t+\varphi)\right] G \\
+\widetilde{C}_{L, \alpha} \hat{\omega}_{y}(k / 2)\left[\sin (\omega t)-\left(k \hat{\alpha}_{y} / 2\right) \cos (\omega t+\varphi)-\hat{\alpha}_{y} \sin (\omega t+\varphi)\right] \\
\hat{\omega}_{y} \equiv \omega y_{A} / V \\
\hat{\alpha}_{y} \equiv \alpha_{A} / \hat{\omega}_{y}
\end{gathered}
$$

Note that $\hat{\omega}_{y}$ is a reduced frequency similar to Theodorsen's ${ }^{7}$ reduced frequency, $k$, except that $\hat{\omega}_{y}$ is based on the aerodynamic-center plunging amplitude, $y_{A}$, whereas $k$ is based on the airfoil section half-chord length, $b$. From Eq. (14), it can be seen that $\hat{\omega}_{y}$ is also the small-angle geometric angle-of-attack amplitude due to the aerodynamiccenter plunging, i.e., amplitude $\left[\dot{h}_{c / 4}(t) / V\right]$. Also notice that $\hat{\alpha}_{y}$ is a geometric angle-of-attack ratio at the airfoil aerodynamic center. It is simply the ratio of the amplitude due to pitching to that due to the aerodynamic-center plunging. Because thrust is produced only by plunging, for flapping flight $\hat{\alpha}_{y}$ is typically small. For the special case when the pitching angle is in phase with the upward quarter-chord $y$-velocity component, Eq. (19) becomes

$$
\begin{aligned}
C_{L}(t) & =\bar{C}_{L}-\widetilde{C}_{L, \alpha} \hat{\omega}_{y}\left(1-\hat{\alpha}_{y}\right)[F \cos (\omega t)-G \sin (\omega t)] \\
& +\widetilde{C}_{L, \alpha} \hat{\omega}_{y}(k / 2)\left\{\left[1-\hat{\alpha}_{y}(1+2 F)\right] \sin (\omega t)-\hat{\alpha}_{y}(2 G+k / 2) \cos (\omega t)\right\}
\end{aligned}
$$

Similarly, Theodorsen's ${ }^{7}$ solution for the traditional quarter-chord pitching-moment coefficient can be obtained from the imaginary part of Eq. (12). Including the effects of camber and finite thickness, this yields

$$
C_{m_{c / 4}}(t) \equiv \frac{\bar{m}_{c / 4}+\operatorname{imag}\left[M_{c / 4}(t)\right]}{\frac{1}{2} \rho V^{2}(2 b)^{2}}=\bar{C}_{m_{c / 4}}-\widetilde{C}_{L, \alpha} \operatorname{imag}\left(\frac{b \ddot{h}_{c / 4}}{8 V^{2}}+\frac{3 b^{2} \ddot{\alpha}}{32 V^{2}}+\frac{b \dot{\alpha}}{4 V}\right)
$$

or after applying Eqs. (13)-(18)

$$
C_{m_{c / 4}}(t)=\bar{C}_{m_{c / 4}}-\widetilde{C}_{L, \alpha} \hat{\omega}_{y}(k / 8)\left[\sin (\omega t)-\left(3 k \hat{\alpha}_{y} / 4\right) \cos (\omega t+\varphi)-2 \hat{\alpha}_{y} \sin (\omega t+\varphi)\right]
$$

For the special case when the pitching angle is in phase with the upward $y$-velocity component, Eq. (23) becomes

$$
C_{m_{c / 4}}(t)=\bar{C}_{m_{c / 4}}-\widetilde{C}_{L, \alpha} \hat{\omega}_{y}(k / 8)\left[\left(1-2 \hat{\alpha}_{y}\right) \sin (\omega t)-\left(3 k \hat{\alpha}_{y} / 4\right) \cos (\omega t)\right]
$$

To compare results predicted from Eqs. (22) and (24) with numerical or experimental results, it is convenient to define the amplitude ratios

$$
\begin{gathered}
R_{A L} \equiv \operatorname{amplitude}\left[C_{L}(t)-\bar{C}_{L}\right] /\left(\widetilde{C}_{L, \alpha} \hat{\omega}_{y}\right) \\
R_{A m} \equiv \operatorname{amplitude}\left[C_{m_{c / 4}}(t)-\bar{C}_{m_{c / 4}}\right] / \widetilde{C}_{L, \alpha} \hat{\omega}_{y}
\end{gathered}
$$


From Eq. (22) we obtain the lift amplitude ratio for the special case when the pitching angle is in phase with the upward quarter-chord $y$-velocity component, i.e.,

$$
R_{A L}=\sqrt{\left[\left(1-\hat{\alpha}_{y}\right) F+\hat{\alpha}_{y}(2 G+k / 2)(k / 2)\right]^{2}+\left[\left(1-\hat{\alpha}_{y}\right) G+(k / 2)-\hat{\alpha}_{y}(1+2 F)(k / 2)\right]^{2}}
$$

The quarter-chord pitching-moment amplitude ratio obtained from Eq. (24) for this same special case is

$$
R_{A m}=(k / 8) \sqrt{\left(1-2 \hat{\alpha}_{y}\right)^{2}+\left(3 k \hat{\alpha}_{y} / 4\right)^{2}}
$$

For low frequencies with $k$ approaching zero, the amplitude ratio predicted from Eq. (27) approaches $\left(1-\hat{\alpha}_{y}\right)$, which is the quasi-steady solution. On the other hand, in the high-frequency limit as $k$ approaches infinity, Eq. (27) predicts infinite oscillations in lift for finite values of $\hat{\omega}_{y}$ and $\hat{\alpha}_{y}$. The high-frequency behavior of Eq. (27) is shown graphically in Fig. 3 plotted as a function of the inverse reduced frequency, $1 / k$, for four different values of the aerodynamic-center geometric angle-of-attack ratio, $\hat{\alpha}_{y}$. This high-frequency limit does not seem realistic.

Similarly, results obtained from Eq. (28) predict that, in the low-frequency limit as $k$ approaches zero, the quarter-chord pitching-moment oscillations vanish, as should be expected for quasi-steady flow. However, in the high-frequency limit as $k$ approaches infinity, the amplitude ratio predicted from Eq. (28) becomes infinite. In other words, the result presented by Theodorsen ${ }^{7}$ predicts that the change in angle of attack induced by the trailing vortex sheet produces an infinite change in the pitching moment about the airfoil section aerodynamic center, for $k$ approaching infinity with finite values of $\hat{\omega}_{y}$ and $\hat{\alpha}_{y}$. High-frequency results obtained from Eq. (28) for the quarterchord pitching-moment amplitude ratio are shown in Fig. 4. Here again, we observe that the high-frequency limit obtained from a result presented by Theodorsen ${ }^{7}$ is unrealistic.

Within the small-angle approximation, the power required to support the plunging is simply the negative of the lift, $L$, multiplied by the $y$ component of the airfoil velocity, $V_{y}$ (positive upward). Similarly, the power required to support the pitching is the negative of the quarter-chord pitching moment, $m_{c / 4}$, multiplied by the quarter-chord angular velocity, $\dot{\alpha}$. Hence, the traditional coefficient for the instantaneous power required is obtained from

$$
C_{P}(t) \equiv \frac{-L(t) V_{y}(t)-m_{c / 4}(t) \dot{\alpha}(t)}{\frac{1}{2} \rho V^{3}(2 b)}=-C_{L}(t) \operatorname{imag}\left(\frac{-\dot{h}_{c / 4}(t)}{V}\right)-C_{m_{c / 4}}(t) \operatorname{imag}\left(\frac{2 b \dot{\alpha}(t)}{V}\right)
$$

After applying Eqs. (14), (17), (22) and, (24), for the special case when the pitching angle, $\alpha(t)$, is in phase with the upward quarter-chord $y$-velocity component, $V_{y}(t)$, the coefficient for the instantaneous power required is obtained from the relation

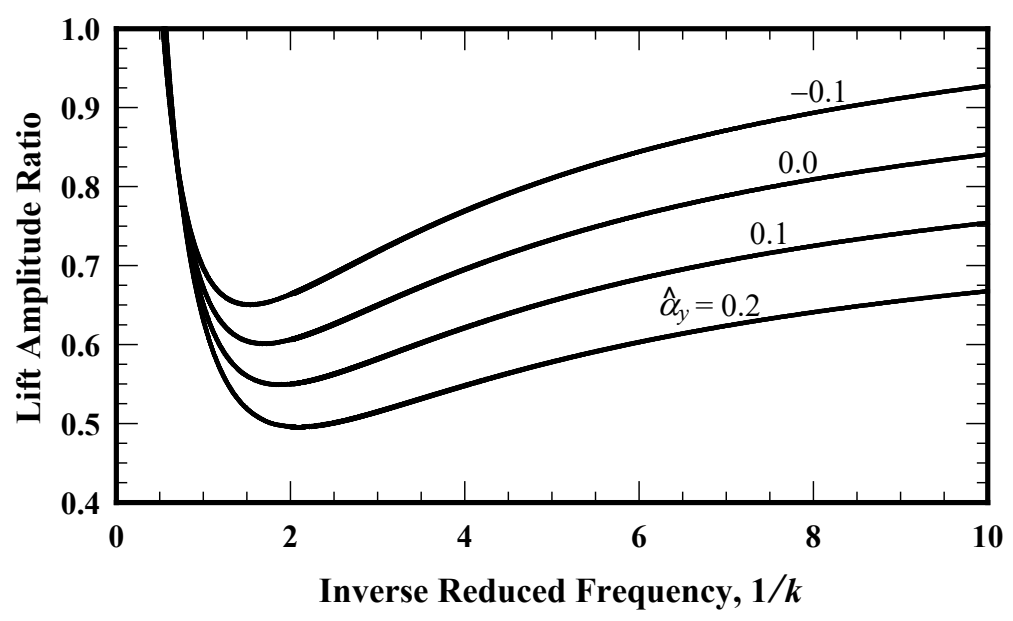

Figure 3. The lift amplitude ratio defined in Eq. (25) as predicted from the results presented by Theodorsen ${ }^{7}$ for a range of aerodynamic-center pitching-to-plunging ratios. 


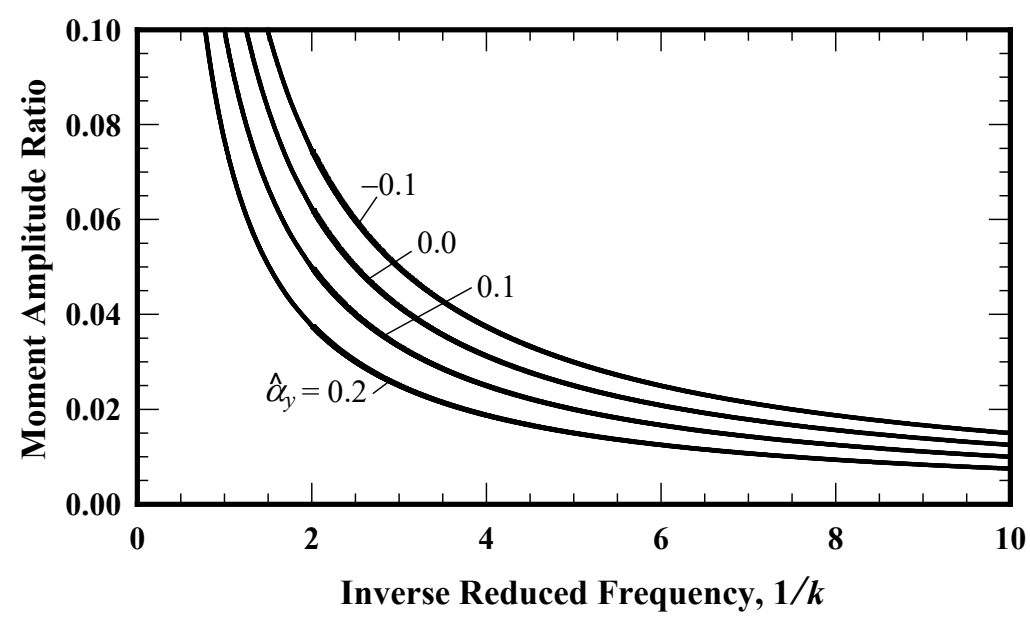

Figure 4. The amplitude ratio defined in Eq. (26) for the quarter-chord pitching moment as predicted from the results presented by Theodorsen ${ }^{7}$ for a range of aerodynamic-center pitching-to-plunging ratios.

$$
\begin{aligned}
C_{P}(t) & =-\left\{\bar{C}_{L} \hat{\omega}_{y}-\widetilde{C}_{L, \alpha} \hat{\omega}_{y}^{2}\left(1-\hat{\alpha}_{y}\right)[F \cos (\omega t)-G \sin (\omega t)]\right\} \cos (\omega t) \\
& -\widetilde{C}_{L, \alpha} \hat{\omega}_{y}^{2}(k / 2)\left\{\left[1-\hat{\alpha}_{y}(1+2 F)\right] \sin (\omega t)-\hat{\alpha}_{y}(2 G+k / 2) \cos (\omega t)\right\} \cos (\omega t) \\
& +\left\{2 \bar{C}_{m_{c / 4}} \hat{\omega}_{y} k \hat{\alpha}_{y}-\widetilde{C}_{L, \alpha} \hat{\omega}_{y}^{2}\left(k^{2} \hat{\alpha}_{y} / 4\right)\left[\left(1-2 \hat{\alpha}_{y}\right) \sin (\omega t)-\left(3 k \hat{\alpha}_{y} / 4\right) \cos (\omega t)\right]\right\} \sin (\omega t)
\end{aligned}
$$

Integrating Eq. (29) over one complete cycle and dividing by the period, $\tau=2 \pi / \omega$, the coefficient for the mean power required is found to be

$$
\bar{C}_{P} \equiv \frac{1}{\tau} \int_{t=0}^{\tau} C_{P}(t) d t=\widetilde{C}_{L, \alpha} \hat{\omega}_{y}^{2}\left[\left(1-\hat{\alpha}_{y}\right) F+k \hat{\alpha}_{y}\left(G+k \hat{\alpha}_{y} / 2\right)\right] / 2
$$

In the low-frequency limit as $k$ approaches zero, the mean power coefficient obtained from Eq. (30) approaches $\widetilde{C}_{L, \alpha} \hat{\omega}_{y}^{2}\left(1-\hat{\alpha}_{y}\right) / 2$, which is the quasi-steady solution. In the high-frequency limit as $k$ approaches infinity, this mean power coefficient approaches $\widetilde{C}_{L, \alpha} \hat{\omega}_{y}^{2}\left(1-\hat{\alpha}_{y}+k^{2} \hat{\alpha}_{y}^{2}\right) / 4$. Results obtained from Eq. (30) for $\bar{C}_{P} /\left(\widetilde{C}_{L, \alpha} \hat{\omega}_{y}^{2}\right)$ are shown in Fig. 5 plotted as a function of the inverse reduced frequency, $1 / k$, for four different values of the aerodynamic-center pitching-to-plunging ratio, $\hat{\alpha}_{y}$. As $k$ approaches infinity, this power coefficient ratio approaches 0.25 for $\hat{\alpha}_{y}=0$. However this high-frequency limit approaches infinity for any nonzero value of $\hat{\alpha}_{y}$. Once again we obtain an unrealistic high-frequency limit from the lift and moment relations presented by Theodorsen, ${ }^{7}$ i.e., an infinite required power predicted from Eq. (30), for $k$ approaching infinity with finite values of $\hat{\omega}_{y}$ and $\hat{\alpha}_{y}$.

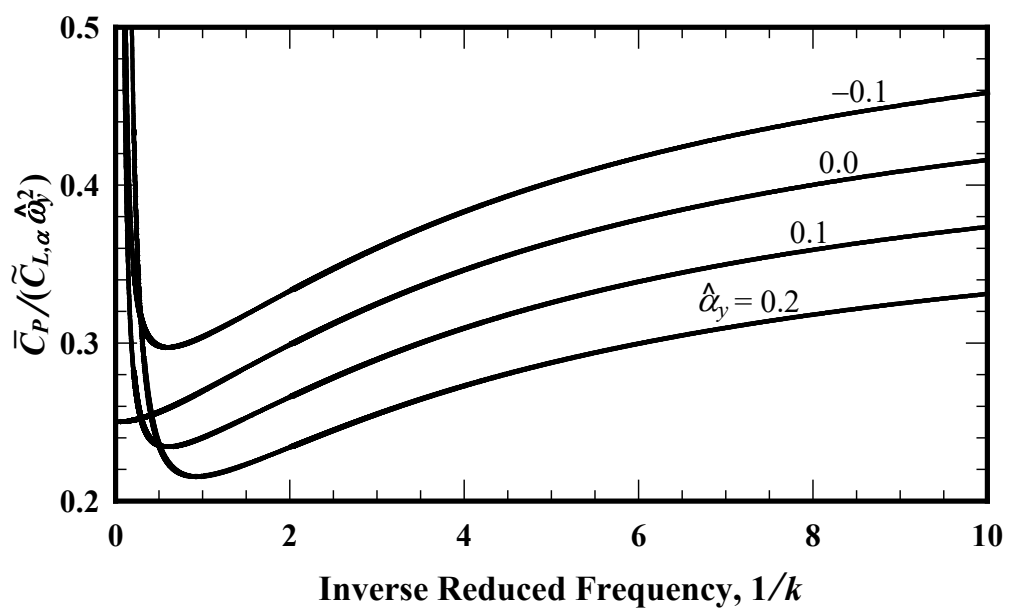

Figure 5. The mean required power coefficient for a range of aerodynamic-center pitching-to-plunging ratios as predicted from Eq. (30), which is obtained from results presented by Theodorsen. ${ }^{7}$ 
In a later NACA report, Garrick ${ }^{8}$ used Theodorsen's 7 model to obtain results for the section "propelling force" for combined sinusoidal plunging and pitching. For the special case when the pitching angle is in phase with the upward quarter-chord $y$-velocity component, Garrick's ${ }^{8}$ solution for the mean section axial-force coefficient is

$$
\bar{C}_{x}=-\widetilde{C}_{L, \alpha} \hat{\omega}_{y}^{2}\left\{\left(F^{2}+G^{2}\right)\left[\left(1-\hat{\alpha}_{y}\right)^{2}+k^{2} \hat{\alpha}_{y}^{2}\right]+F\left(\hat{\alpha}_{y}-\hat{\alpha}_{y}^{2}-k^{2} \hat{\alpha}_{y}^{2}\right)+k \hat{\alpha}_{y}\left(G+k \hat{\alpha}_{y} / 2\right)\right\} / 2
$$

In the low-frequency limit as $k$ approaches zero, the mean axial-force coefficient predicted from Eq. (31) approaches the quasi-steady solution, $-\widetilde{C}_{L, \alpha} \hat{\omega}_{y}^{2}\left(1-\hat{\alpha}_{y}\right) / 2$. As $k$ goes to infinity, this mean axial-force coefficient approaches $-\widetilde{C}_{L, \alpha} \hat{\omega}_{y}^{2}\left(1-\hat{\alpha}_{y}^{2}+k^{2} \hat{\alpha}_{y}^{2}\right) / 8$. High-frequency results from Eq. (31) are shown in Fig. 6 plotted as a function of the inverse reduced frequency, $1 / k$, for four different values of the aerodynamic-center pitching-to-plunging ratio, $\hat{\alpha}_{y}$. Again, the high-frequency limit of this solution is unrealistic for finite values of $\hat{\omega}_{y}$ and $\hat{\alpha}_{y}$.

The instantaneous propulsive power available is simply the negative of the $x$ component of the aerodynamic force acting on the airfoil, $F_{x}$, multiplied by the forward airspeed, $V$. Hence, the traditional aerodynamic coefficient for the propulsive power available is simply

$$
\frac{-F_{x} V}{\frac{1}{2} \rho V^{3}(2 b)}=-C_{x}(t)
$$

The propulsive efficiency is the mean propulsive power available divided by the mean power required. Hence, in view of Eqs. (30), (31), and (32), the propulsive efficiency obtained from Theodorsen's ${ }^{7}$ model for the special case when the pitching angle, $\alpha(t)$, is in phase with the upward quarter-chord $y$-velocity component, $V_{y}(t)$, is given by

$$
\eta=\frac{-\bar{C}_{x}}{\bar{C}_{P}}=\frac{\left(F^{2}+G^{2}\right)\left[\left(1-\hat{\alpha}_{y}\right)^{2}+k^{2} \hat{\alpha}_{y}^{2}\right]+F\left(\hat{\alpha}_{y}-\hat{\alpha}_{y}^{2}-k^{2} \hat{\alpha}_{y}^{2}\right)+k \hat{\alpha}_{y}\left(G+k \hat{\alpha}_{y} / 2\right)}{\left(1-\hat{\alpha}_{y}\right) F+k \hat{\alpha}_{y}\left(G+k \hat{\alpha}_{y} / 2\right)}
$$

In the low-frequency limit as $k$ approaches zero, the propulsive efficiency obtained from Eq. (33) approaches 1.0, as expected for the quasi-steady solution. As $k$ approaches infinity, this propulsive efficiency approaches 0.5 . Highfrequency results for the propulsive efficiency obtained from Eq. (33) are plotted in Fig. 7 as a function of the inverse reduced frequency, $1 / k$, for four different values of the aerodynamic-center pitching-to-plunging ratio, $\hat{\alpha}_{y}$.

The high-frequency results obtained from the model by Theodorsen ${ }^{7}$ and shown in Figs. 3-6 are clearly unrealistic. However, the model exhibits the correct asymptotic behavior at low frequencies. It is therefore important to assess the accuracy of this model in the range of interest for any application before applying it to a simulation. Here we examine the accuracy of this model over the range of flapping frequencies commonly encountered in the forward flight of birds. Typical reduced frequencies for birds in forward flight range between

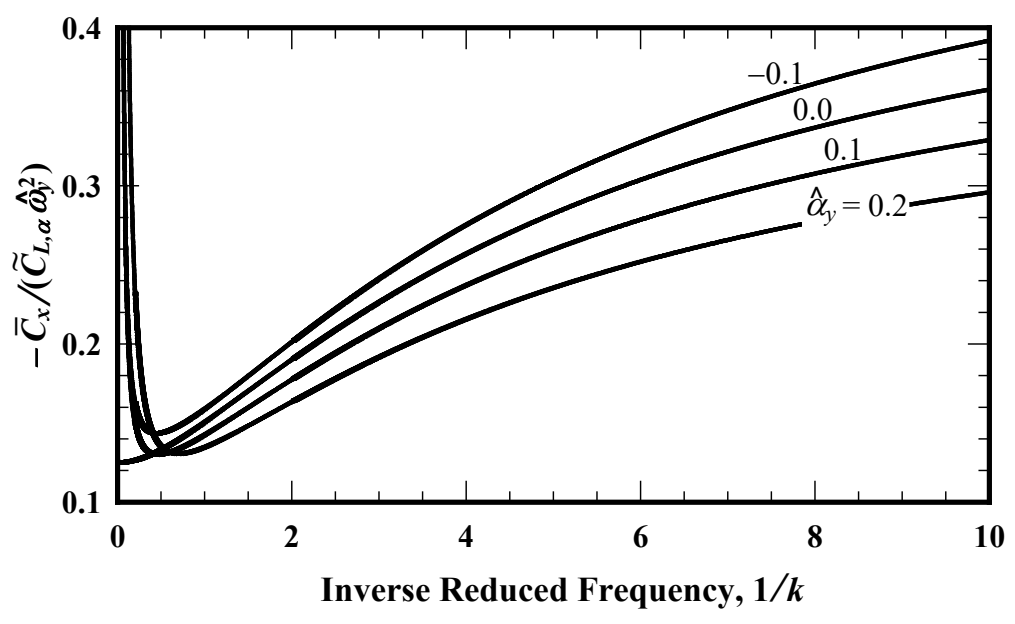

Figure 6. The mean axial-force coefficient for a range of aerodynamic-center pitching-to-plunging ratios as predicted from Eq. (31), which is obtained from results presented by Garrick. ${ }^{8}$ 


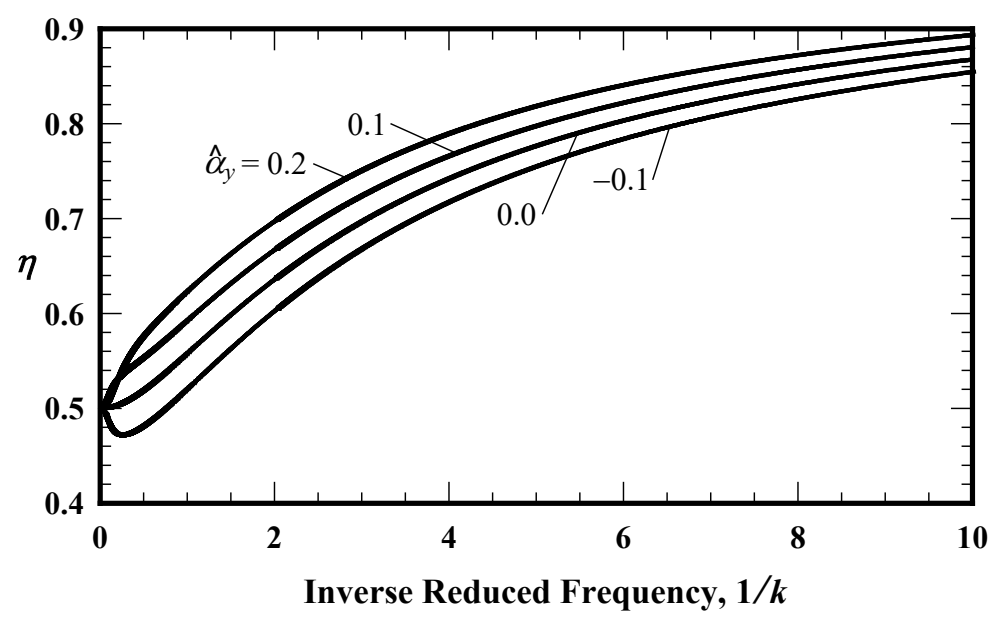

Figure 7. The propulsive efficiency for a range of aerodynamic-center pitching-to-plunging ratios as predicted from Eq. (33), which is obtained from results presented by Theodorsen ${ }^{7}$ and Garrick. $^{8}$

$k \cong 0.015$ for low-frequency flapping birds such as the wandering albatross to $k \cong 0.2$ for high-frequency flapping birds such as the ruby-throated hummingbird. In terms of the traditional plots presented by Theodorsen, this gives a range of $5 \approx 1 / k \approx 70$. In order to evaluate the accuracy of the Theodorsen model within this frequency range, a large number of CFD simulations were performed. A detailed description of this process is given in the following section.

\section{Comparison with Inviscid Computational Fluid Dynamics Solutions}

All CFD solutions were obtained using version 8.06 of the commercially available software Star-CCM.$+{ }^{57}$ This software is capable of solving the three-dimensional, time-dependent, Reynolds-averaged, Navier-Stokes equations using a finite-volume formulation on an unstructured grid. However, to be consistent with potential flow theory upon which Theodorsen's ${ }^{7}$ model is based, the available inviscid solver was used for this study. Unsteady, incompressible computations were performed using the segregated flow solver. Second-order upwinding was used for the convection terms and the implicit unsteady solver was used with second-order temporal discretization. Constant fluid density of $1.225 \mathrm{~kg} / \mathrm{m}^{3}$ and a freestream velocity of $14 \mathrm{~m} / \mathrm{s}$ were used for all computations, which gives a freestream Mach number of 0.041. A von Kármán Trefftz airfoil with a maximum thickness of $15 \%$, a chord length of $0.25 \mathrm{~m}$, and a zero-lift angle of attack of -5.0 degrees was used for these computations. This airfoil was chosen because it has a closed-form analytical solution for steady flow, which was used to evaluate the error in the grid convergence study. The analytical aerodynamic center of this airfoil is located at $x / c=0.2757$ and $y / c=0.0061$.

The time-dependent motion of the airfoil was modeled using an overset grid that moved relative to a fixed background grid. The overset grid translated vertically according to the aerodynamic-center plunging motion given in Eq. (13), $y_{A} \sin (\omega t)$, while rotating about the airfoil aerodynamic center according to the pitching motion given in Eq. (16), $\alpha_{A} \cos (\omega t)$. The pitching and plunging motions were specified by the pitching and plunging velocities, which were integrated by the software to evaluate the position and orientation as a function of time.

A structured C-grid was used for both the overset and background grids. Nodes were clustered near the leading and trailing edges of the airfoil and in the wake region aft of the airfoil to improve accuracy and provide improved resolution of the oscillating wake. ${ }^{58}$ The C-portion of the background grid was set as a velocity inlet boundary, the exit plane was set as a flow-split outlet with a split ratio of 1.0, and a slip boundary condition was specified on the airfoil surface. Two-dimensional airfoil results were obtained from the three-dimensional solver by using three identical grid planes in the $z$-direction, and setting the two outboard $z$-planes to symmetry boundary conditions.

To evaluate grid convergence, a family of three similar grids was generated from an initial fine background grid. A medium background grid was generated by removing every other node from the fine grid, and a coarse background grid was created by removing every other node from the medium grid. The overset grid for each case was generated by removing several outer circumferential layers from the background grid. A sample coarse grid can be seen in Fig. 8. 


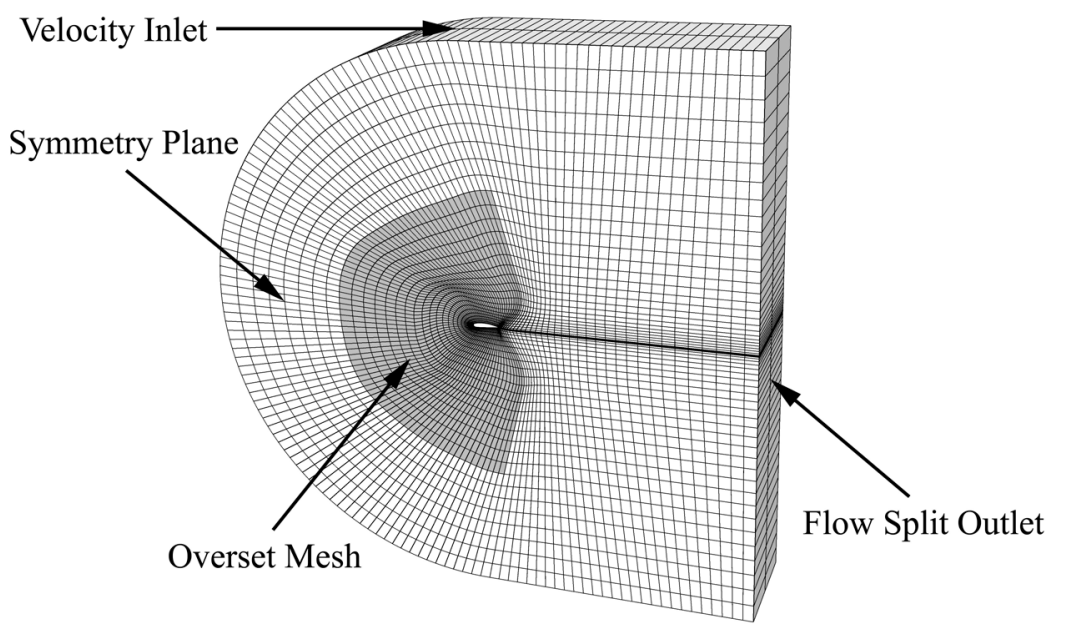

Figure 8. Example coarse grid for computational fluid dynamics calculations.

To evaluate the accuracy of solutions obtained from a given grid, results from a series of steady-flow cases representing 16 steps in a quasi-steady flapping cycle were compared with the analytical solution for the airfoil forces and pitching moment. For each case, the freestream angle of attack was set to zero, the overset grid was offset relative to the background grid according to the translation specified by Eq. (13), $y_{A} \sin (\omega t)$, and the overset grid was given a steady translational velocity specified by Eq. (14), $\omega y_{A} \cos (\omega t)$. This created a steady-state case at the same vertical offset and geometric angle of attack as the airfoil would experience at that point in the transient simulations. Because inviscid CFD computations usually produce flow separation at lower angles of attack than are observed experimentally, values for $y_{A}$ and $\omega$ used for this grid convergence study were chosen to give geometric angles of attack ranging from -4.0 to 4.0 degrees. This gives a range in the analytical lift coefficient from 0.1232 to 1.104. The grid was refined and the size of the overset grid was varied to reduce the CFD error, relative to the analytical solutions, to an acceptable level. These grid refinement studies resulted in a final fine background grid with 561 circumferential nodes and 121 radial nodes (67,200 cells per plane) and an overset grid with 337 circumferential nodes and 89 radial nodes (29,568 cells per plane). This grid will be referred to here as the fine grid.

Figures 9-11 show typical results for the lift, drag, and aerodynamic-center pitching-moment coefficients for the final coarse, medium, and fine grids compared to the analytical solutions in the angle-of-attack range from -4.0 to 4.0 degrees. The fine-grid error in the lift and aerodynamic-center pitching-moment coefficients was less than $0.5 \%$ of the mean values. The finite drag predicted by the CFD solutions and shown in Fig. 10 is due to numerical viscosity and results in a mean fine-grid drag-coefficient error of 0.00031 in this angles-of-attack range. To ensure that the computational domain size was sufficient, the outer radius of the background C-grid was increased from 10 chord lengths to 20 chord lengths for a representative subset of cases with insignificant change in the results. Based on the results of this grid convergence study, the fine $561 \times 121$-node grid was used for all solutions presented here.

As mentioned earlier, typical axial reduced frequencies for birds in forward flight range between $k \cong 0.015$ to $k \cong 0.2$. Additionally, in order to prevent large numerical viscosity errors and the associated flow separation with inviscid CFD calculations, the geometric angle of attack must be typically limited to the range \pm 6.0 degrees. Therefore, for all unsteady cases presented here, values for $y_{A}, \omega$, and $\alpha_{A}$ were chosen such that the geometric angle of attack fell in the range $-5.37<\alpha_{g}<5.37$ degrees. This geometric-angle-of-attack constraint combined with the fact that the vertical displacement of the overset grid is limited resulted in a feasible reduced-frequency range of $0.05<k<0.42$.

All transient CFD solutions were obtained using 128 time steps per cycle for eight cycles. The convergence criterion at each time step was set so that the difference in the $y$-momentum residual over 100 iterations was less than $10^{-8}$. This criterion was set to $10^{-15}$ for a representative subset of cases with no significant change in the results. To assure that steady periodic solutions were obtained, the values of the axial force, normal force, and aerodynamiccenter pitching moment at each time step during cycle eight were compared to the corresponding values during cycle seven. The maximum instantaneous deviation between the seventh and eighth cycle in percent of the mean ranged from $1.0 \times 10^{-11 \%}$ to $2.6 \times 10^{-2} \%$ for the axial-force coefficient, from $3.0 \times 10^{-12} \%$ to $3.8 \times 10^{-3} \%$ for the normalforce coefficient, and from $2.3 \times 10^{-12 \%}$ to $1.8 \times 10^{-4} \%$ for the aerodynamic-center pitching-moment coefficient. 


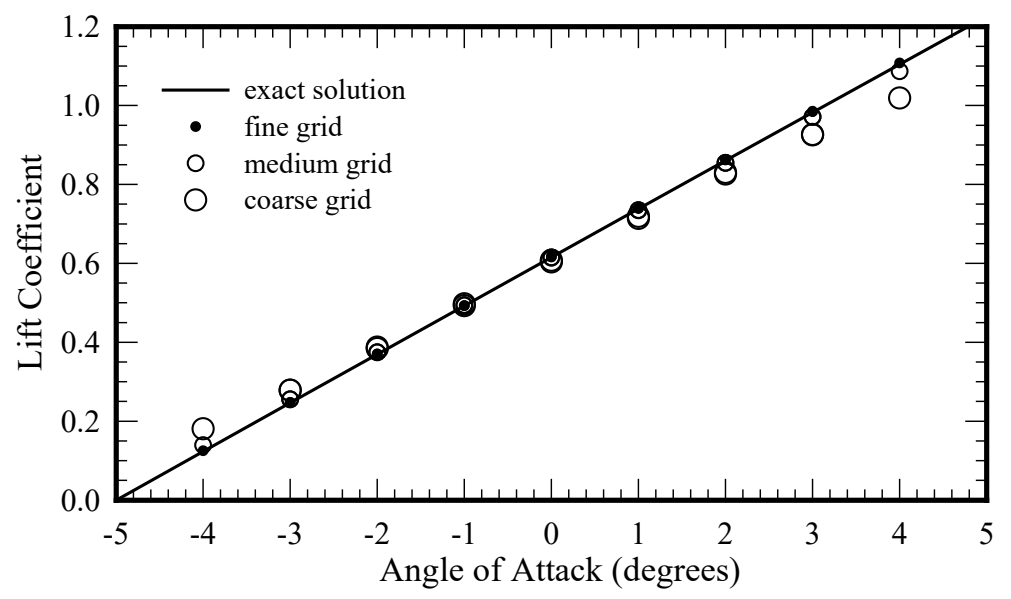

Figure 9. Coarse-, medium-, and fine-grid CFD solutions for the steady lift coefficient compared with the exact analytical solution.

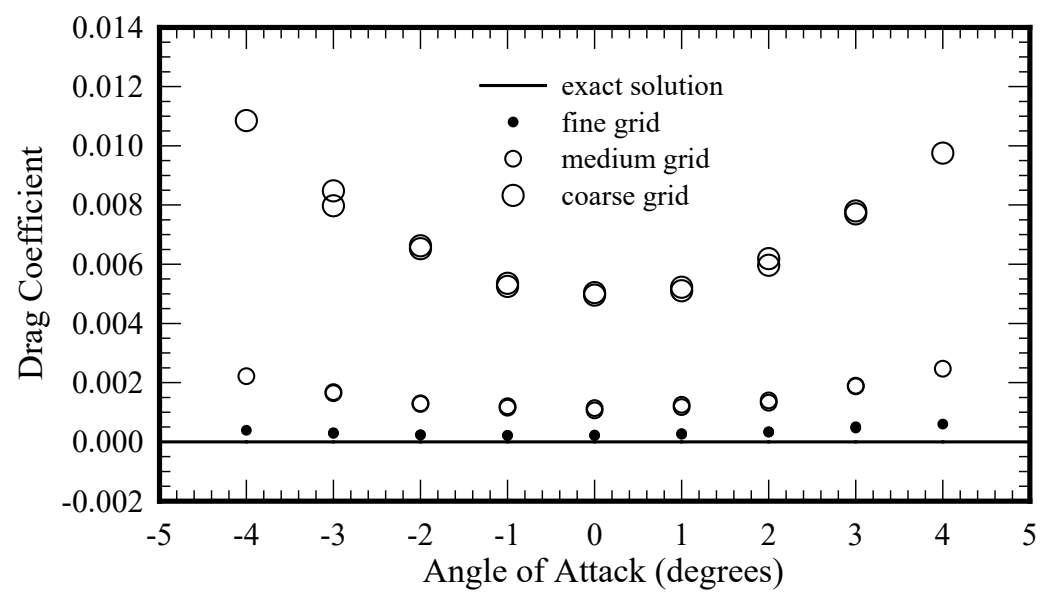

Figure 10. Coarse-, medium-, and fine-grid CFD solutions for the steady drag coefficient compared with the exact analytical solution.

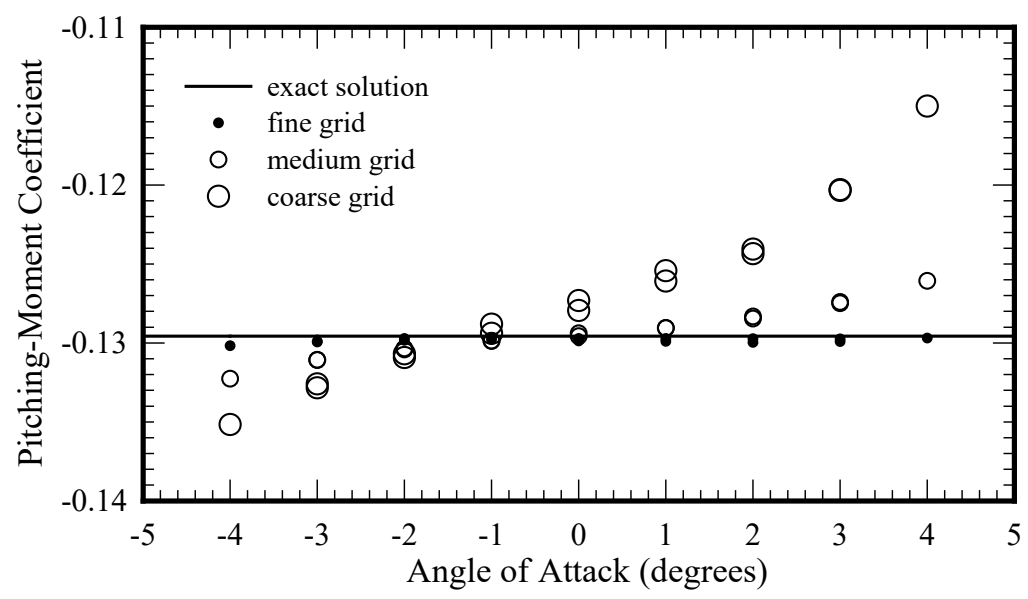

Figure 11. Coarse-, medium-, and fine-grid CFD solutions for the steady pitching-moment coefficient about the airfoil aerodynamic center compared with the exact analytical solution. 
To assure that these CFD solutions were time-step converged, solutions were repeated using 256 time steps per cycle for a representative subset of the solutions. The mean deviation in the eighth cycle between the 128-time-step solutions and the 256 -time-step solutions ranged from $0.03 \%$ to $0.54 \%$ for the axial-force coefficient, from $0.0025 \%$ to $0.0055 \%$ for the normal-force coefficient, and from $0.0004 \%$ to $0.0046 \%$ for the aerodynamic-center pitchingmoment coefficient. From the analysis described above, it was concluded that the steady periodic CFD solutions obtained using the $561 \times 121$-node grid with 128 time steps per cycle for the duration of eight cycles were all grid and time-step converged.

From each CFD solution, values were obtained for the mean axial-force coefficient and the mean requiredpower coefficient. Figures 12 and 13 show typical CFD results compared to results for the Theodorsen ${ }^{7}$ model over the range of reduced frequencies included in Fig. 2, but using a logarithmic scale for the $x$-axis so that the highfrequency range (low values for $1 / k$ ) is more visible. All results shown in Figs. 12 and 13 were obtained for a geometric angle-of-attack amplitude of $\hat{\omega}_{y}\left(1-\hat{\alpha}_{y}\right)=4.29^{\circ}$ and a mean lift coefficient of $\bar{C}_{L}=0.614$. The deviation between the CFD results and Eqs. (30), (31), and (33) for all cases in this study ranges from $-2.9 \%$ to $34 \%$ for the mean axial-force coefficient, from $-1.2 \%$ to $24 \%$ for the mean required-power coefficient, and from $-2.4 \%$ to $8.3 \%$ for the propulsive efficiency. Note that, in general, the error for each coefficient increases with increasing reduced frequency. For reduced frequencies in the range $k<0.1$ or $1 / k>10$, the Theodorsen model agrees with all computational results in this study to within $10 \%$. Even at higher reduced frequencies, the Theodorsen model agrees with the CFD results to within $35 \%$. This is rather remarkable considering the seemingly constraining approximations used by Theodorsen in developing the model.

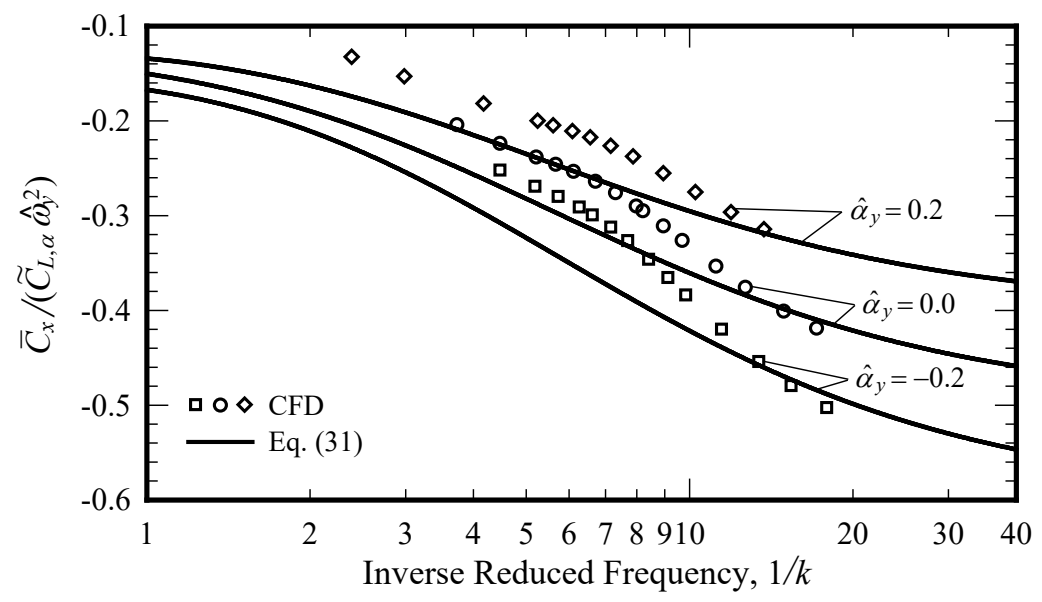

Figure 12. Comparison between the CFD solutions and Eq. (31).

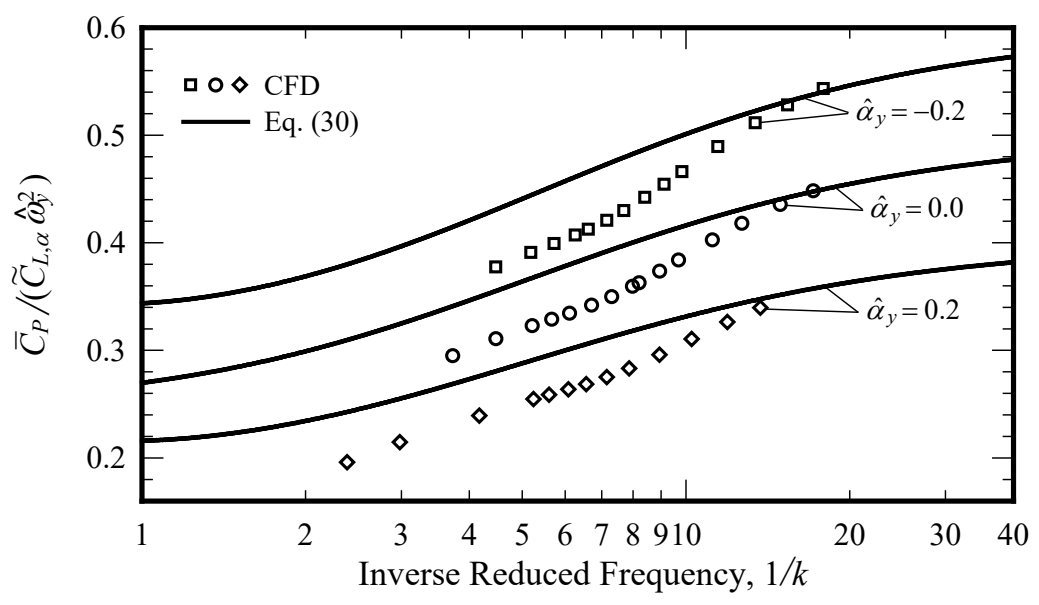

Figure 13. Comparison between the CFD solutions and Eq. (30). 


\section{An Alternate Flapping-Airfoil Propulsion Model}

An alternate approach to that taken by Theodorsen ${ }^{7}$ for predicting the aerodynamics of a flapping airfoil is to use numerical solutions or experimental data to calibrate the frequency dependence of the unsteady aerodynamic coefficients. The CFD solutions produced in this work and discussed in the previous section provide a dataset that can be used for this purpose. Such an approach may be better suited for modeling the propulsion of flapping airfoils at high flapping frequencies where Theodorsen's model is less accurate. This approach is illustrated in this section, and algebraic relations are presented that can be used to predict the thrust, required power, and propulsive efficiency of flapping airfoils over a wide range of flapping frequencies.

Consider a 2-D airfoil section immersed in a uniform flow with a constant freestream velocity, $V$. The airfoil is undergoing sinusoidal vertical displacement, $y_{a c}(t)$, of its aerodynamic center combined with small-angle sinusoidal rotation, $\alpha(t)$, about its aerodynamic center. The displacement $y_{a c}$ has a zero mean and is positive upward, the rotation $\alpha$ has a mean value of $\bar{\alpha}$ and is positive clockwise relative to the constant freestream velocity, which approaches the airfoil from the left. For this motion, the position of the airfoil's aerodynamic center in the direction normal to the freestream velocity can be expressed in terms of an amplitude, $y_{A}$, and a frequency, $\omega$, i.e.,

$$
y_{a c}(t)=y_{A} \sin (\omega t)
$$

Hence, the upward normal-velocity component of the airfoil's aerodynamic center is

$$
V_{y}(t)=\omega y_{A} \cos (\omega t)
$$

Here we shall consider the case when the pitching angle, $\alpha(t)$, is oscillating at the same frequency and is in phase with the upward $y$-velocity component, $V_{y}(t)$. Denoting the pitching amplitude as $\alpha_{A}$, we have

$$
\alpha(t)=\bar{\alpha}+\alpha_{A} \cos (\omega t)
$$

Within the small-angle approximation, the total geometric angle of attack is then

$$
\alpha_{g}(t)=\bar{\alpha}-\hat{\omega}_{y}\left(1-\hat{\alpha}_{y}\right) \cos (\omega t)
$$

where $\hat{\omega}_{y}$ and $\hat{\alpha}_{y}$ are defined in Eqs. (20) and (21) respectively.

Because the trailing vortex sheet is time dependent, there is also a time-dependent induced angle of attack, $\alpha_{i}(t)$, and the lift coefficient can be written in terms of the airfoil section lift slope $\widetilde{C}_{L, \alpha}$ and the zero-lift angle of attack $\alpha_{L 0}$ as

$$
C_{L}(t)=\widetilde{C}_{L, \alpha}\left[\alpha_{g}(t)+\alpha_{i}(t)-\alpha_{L 0}\right]
$$

or after applying Eq. (37),

$$
C_{L}(t)=\widetilde{C}_{L, \alpha}\left[\bar{\alpha}-\hat{\omega}_{y}\left(1-\hat{\alpha}_{y}\right) \cos (\omega t)+\alpha_{i}(t)-\alpha_{L 0}\right]
$$

The lift vector is perpendicular to the relative wind. Hence, it is rotated through the aerodynamic-center angle of attack due to plunging, $-\hat{\omega}_{y} \cos (\omega t)$, and the induced angle of attack, $\alpha_{i}(t)$. However, it is not rotated through the angle of attack due to pitching. Continuing with the small-angle approximation, the axial and normal components of lift are

$$
\begin{gathered}
C_{x}(t)=-\widetilde{C}_{L, \alpha}\left[\bar{\alpha}-\hat{\omega}_{y}\left(1-\hat{\alpha}_{y}\right) \cos (\omega t)+\alpha_{i}(t)-\alpha_{L 0}\right]\left[-\hat{\omega}_{y} \cos (\omega t)+\alpha_{i}(t)\right] \\
C_{y}(t)=C_{L}(t)=\widetilde{C}_{L, \alpha}\left[\bar{\alpha}-\hat{\omega}_{y}\left(1-\hat{\alpha}_{y}\right) \cos (\omega t)+\alpha_{i}(t)-\alpha_{L 0}\right]
\end{gathered}
$$

In general, the instantaneous induced angle of attack could depend on the oscillating component of the geometric angle of attack and all of its time derivatives, i.e., 


$$
\alpha_{i}(t)=C_{0}\left[\alpha_{g}(t)-\bar{\alpha}\right]+\sum_{n=1}^{\infty} C_{n} \frac{d^{n} \alpha_{g}}{d t^{n}}
$$

The time derivatives of the geometric angle of attack can be evaluated from Eq. (37), which gives

$$
\frac{d^{n} \alpha_{g}}{d t^{n}}=\hat{\omega}_{y}\left(1-\hat{\alpha}_{y}\right)\left\{\begin{array}{l}
(-1)^{(n+3) / 2} \omega^{n} \sin (\omega t), \text { for } n \text { odd } \\
(-1)^{(n+2) / 2} \omega^{n} \cos (\omega t), \text { for } n \text { even }
\end{array}\right.
$$

Using Eqs. (37) and (43) in Eq. (42) yields

$$
\begin{aligned}
& \alpha_{i}(t)=\hat{\omega}_{y}\left(1-\hat{\alpha}_{y}\right)[A \cos (\omega t)+B \sin (\omega t)], \text { where } \\
& A \equiv \sum_{\substack{n=0 \\
n \text { even }}}^{\infty}(-1)^{(n+2) / 2} C_{n} \omega^{n}, \quad B \equiv \sum_{\substack{n=1 \\
n \text { odd }}}^{\infty}(-1)^{(n+3) / 2} C_{n} \omega^{n}
\end{aligned}
$$

Note that the unknown coefficients $A$ and $B$ in Eq. (44) depend on the sinusoidal frequency. Furthermore, the coefficients $C_{n}$ could depend on the frequency and other flow-field variables, such as the freestream velocity, airfoil chord length, and the plunging and pitching amplitudes. However, for this steady sinusoidal plunging and pitching, the coefficients $A$ and $B$ do not vary with time over the flapping cycle.

Applying the trigonometric identities $\cos ^{2} \theta=[1+\square \cos (2 \theta)] / 2, \sin ^{2} \theta=[1-\cos (2 \theta)] / 2$, and $\cos \theta \sin \theta=\sin (2 \theta) / 2$, while using Eq. (44) in Eqs. (40) and (41), the instantaneous axial- and normal-force coefficients are found to be

$$
\begin{gathered}
C_{x}(t)=\bar{C}_{L} \hat{\omega}_{y}\left[A_{x 1} \cos (\omega t)+B_{x 1} \sin (\omega t)\right]-\widetilde{C}_{L, \alpha} \hat{\omega}_{y}^{2}\left(1-\hat{\alpha}_{y}\right)\left[A_{x 0}+A_{x 2} \cos (2 \omega t)+B_{x 2} \sin (2 \omega t)\right] \\
A_{x 0} \equiv\left[(1-A)\left(1-A+\hat{\alpha}_{y} A\right)+\left(1-\hat{\alpha}_{y}\right) B^{2}\right] / 2, \quad A_{x 1} \equiv 1-\left(1-\hat{\alpha}_{y}\right) A, \quad B_{x 1} \equiv-\left(1-\hat{\alpha}_{y}\right) B, \\
A_{x 2} \equiv\left[(1-A)\left(1-A+\hat{\alpha}_{y} A\right)-\left(1-\hat{\alpha}_{y}\right) B^{2}\right] / 2, \quad B_{x 2} \equiv-\left[(1-A)\left(1-\hat{\alpha}_{y}\right)+\hat{\alpha}_{y} / 2\right] B \\
C_{y}(t)=\bar{C}_{L}-\widetilde{C}_{L, \alpha} \hat{\omega}_{y}\left(1-\hat{\alpha}_{y}\right)\left[A_{y 1} \cos (\omega t)+B_{y 1} \sin (\omega t)\right] \\
A_{y 1} \equiv 1-A, \quad B_{y 1} \equiv-B
\end{gathered}
$$

where the mean lift coefficient is

$$
\bar{C}_{L}=\widetilde{C}_{L, \alpha}\left(\bar{\alpha}-\alpha_{L 0}\right)
$$

Integrating Eq. (45) over one cycle and dividing by the period, $\tau=2 \pi / \omega$, the mean axial-force coefficient is

$$
\bar{C}_{x} \equiv \frac{1}{\tau} \int_{t=0}^{\tau} C_{x}(t) d t=-\widetilde{C}_{L, \alpha} \hat{\omega}_{y}^{2}\left(1-\hat{\alpha}_{y}\right)\left[(1-A)\left(1-A+\hat{\alpha}_{y} A\right)+\left(1-\hat{\alpha}_{y}\right) B^{2}\right] / 2
$$

Each pair of sinusoidal terms in Eqs. (45) and (46) can be written in terms of an amplitude and phase. For example, the pair of sinusoidal terms in Eq. (46) can be written as

$$
A_{y 1} \cos (\omega t)+B_{y 1} \sin (\omega t)=R_{y 1} \cos \left(\omega t+\varphi_{y 1}\right)
$$

where

$$
R_{y 1}=\sqrt{A_{y 1}^{2}+B_{y 1}^{2}}=\sqrt{(1-A)^{2}+B^{2}}
$$




$$
\varphi_{y 1}=\tan ^{-1}\left(-B_{y 1} / A_{y 1}\right)=\tan ^{-1}[B /(1-A)]
$$

Notice from Eqs. (44)-(46) that the 7 coefficients, $A_{x 0}, A_{x 1}, B_{x 1}, A_{x 2}, B_{x 2}, A_{y 1}$, and $B_{y 1}$, depend on the plunging frequency. However, for steady sinusoidal oscillations, these are Fourier coefficients that do not vary with time over the flapping cycle. It should also be noted that, within the small-angle approximation, the normal-force coefficient varies sinusoidally with time at the plunging frequency, but is phase shifted relative to the plunging cycle. The temporal variation in the axial-force coefficient oscillates with only the plunging frequency and its first harmonic, both of which are phase shifted relative to the plunging cycle.

From Eqs. (45) and (46), we see that the axial- and normal-force coefficients depend on at least one important reduced frequency, $\hat{\omega}_{y}$, which was defined in Eq. (20). Traditionally, the term reduced frequency is used to denote a dimensionless parameter defined to be the product of a frequency and a characteristic length divided by the freestream airspeed. There are two important characteristic lengths associated with steady sinusoidal oscillation of an airfoil. The characteristic length in the axial direction is the chord length, $c$, and that in the normal direction is the aerodynamic-center plunging amplitude, $y_{A}$. Hence, we should expect the axial- and normal-force coefficients to depend on two reduced frequencies, i.e., the normal reduced frequency, $\hat{\omega}_{y}$, and an axial reduced frequency,

$$
\hat{\omega}_{x} \equiv \omega c / V
$$

The reduced frequency $\hat{\omega}_{y}$ appears naturally in the formulation presented here. The reduced frequency $\hat{\omega}_{x}$ affects the solution through its effect on the time-dependent trailing vortex sheet. Because the instantaneous induced angle of attack depends on the variation in the strength of the trailing vortex sheet with the axial coordinate, we should expect the Fourier coefficients $A$ and $B$, defined in Eq. (44), to be strong functions of $\hat{\omega}_{x}$. It should be noted that $\hat{\omega}_{x}$ is simply twice the reduced frequency introduced by Theodorsen ${ }^{7}$, ie., $\hat{\omega}_{x}=2 k$. This is because we have chosen the chord length as the axial length scale, whereas Theodorsen's ${ }^{7}$ reduced frequency was based on the half-chord length.

For the special case of quasi-steady sinusoidal oscillations, the axial reduced frequency $\hat{\omega}_{x}$, the induced angle of attack $\alpha_{i}$, and the Fourier coefficients $A$ and $B$ all approach zero. Hence, the 7 Fourier coefficients in Eqs. (45) and (46) reduce to the low-frequency limit

$$
A_{x 0}=1 / 2, \quad A_{x 1}=1, \quad B_{x 1}=0, \quad A_{x 2}=1 / 2, \quad B_{x 2}=0, \quad A_{y 1}=1, \quad B_{y 1}=0
$$

The traditional coefficient for the instantaneous power required to support this pitching and plunging motion is

$$
C_{P}(t) \equiv \frac{-F_{y}(t) V_{y}(t)-m_{a c} \dot{\alpha}(t)}{\frac{1}{2} \rho V^{3} c}=\frac{-C_{y}(t) V_{y}(t)-C_{m_{a c}} c \dot{\alpha}(t)}{V}
$$

Within the small-angle approximation, for the special case when the pitching angle is in phase with the upward $y$ velocity component of the airfoil's aerodynamic center, after applying Eqs. (20), (21), (35), (36), (46), and (52), the coefficient for the instantaneous power required is obtained from the relation

$$
C_{P}(t)=-\left\{\bar{C}_{L} \hat{\omega}_{y}-\widetilde{C}_{L, \alpha} \hat{\omega}_{y}^{2}\left(1-\hat{\alpha}_{y}\right)[(1-A) \cos (\omega t)-B \sin (\omega t)]\right\} \cos (\omega t)+C_{m_{a c}} \hat{\omega}_{x} \hat{\omega}_{y} \hat{\alpha}_{y} \sin (\omega t)
$$

Integrating Eq. (53) over one cycle and dividing by the period, $\tau=2 \pi / \omega$, the mean required-power coefficient is

$$
\bar{C}_{P} \equiv \frac{1}{\tau} \int_{t=0}^{\tau} C_{P}(t) d t=\widetilde{C}_{L, \alpha} \hat{\omega}_{y}^{2}\left(1-\hat{\alpha}_{y}\right)(1-A) / 2
$$

The traditional aerodynamic coefficient for the available propulsive power is given in Eq. (32). The propulsive efficiency is the mean available propulsive power divided by the mean power required. Hence, in view of Eqs. (48), (54), and (32), for the special case when the pitching angle is in phase with the upward $y$-velocity component of the airfoil's aerodynamic center, the propulsive efficiency is given by

$$
\eta=-\bar{C}_{x} / \bar{C}_{P}=1-\left(1-\hat{\alpha}_{y}\right)\left[A-B^{2} /(1-A)\right]
$$


In order to predict the time-dependent wake-induced angle of attack and its effect on the airfoil forces, we must obtain values for the Fourier coefficients $A$ and $B$, which were introduced in Eq. (44). Notice from Eq. (54) that, within the small-angle approximation, the mean required power is independent of the Fourier coefficient $B$. Hence, if the mean required-power coefficient is somehow determined for a given set of operating conditions, the corresponding Fourier coefficient $A$ can be evaluated from Eq. (54), i.e.,

$$
A=1-\frac{2 \bar{C}_{P}}{\widetilde{C}_{L, \alpha} \hat{\omega}_{y}^{2}\left(1-\hat{\alpha}_{y}\right)}
$$

Furthermore, if the mean axial-force coefficient is similarly determined, the Fourier coefficient $B$ can be evaluated from Eq. (48) and the known value of $A$ that was obtained from the power coefficient using Eq. (56). This gives

$$
B=\sqrt{\frac{-2 \bar{C}_{x}}{\widetilde{C}_{L, \alpha} \hat{\omega}_{y}^{2}\left(1-\hat{\alpha}_{y}\right)^{2}}-\frac{(1-A)\left(1-A+\hat{\alpha}_{y} A\right)}{\left(1-\hat{\alpha}_{y}\right)}}
$$

Once the Fourier coefficients $A$ and $B$ have been determined from Eqs. (56) and (57), the temporal variation in the wake-induced angle of attack can be obtained from Eq. (44).

The mean required-power and axial-force coefficients were evaluated for a wide range of operating conditions from the inviscid CFD solutions presented in the previous section. For these computations, the axial reduced frequency $\hat{\omega}_{x}$ was varied from 0.10 to 0.84 ; the vertical reduced frequency $\hat{\omega}_{y}$ was varied from 0.025 to 0.117 ; and the aerodynamic-center pitching-to-plunging ratio $\hat{\alpha}_{y}$ was varied from -0.2 to 0.2 . These solutions were used with Eqs. (56) and (57) to evaluate the Fourier coefficients $A$ and $B$ and the results were used to obtain algebraic correlation equations, which relate $A$ and $B$ to the nondimensional operating parameters $\hat{\omega}_{x}, \hat{\omega}_{y}$, and $\hat{\alpha}_{y}$. It was found that the values for the Fourier coefficients $A$ and $B$ obtained from these CFD solutions could be correlated quite accurately using the following piecewise continuous functions:

$$
\begin{aligned}
& A=\left(1+0.13 \hat{\alpha}_{y}\right)\left\{\begin{array}{l}
A_{15} \hat{\omega}_{x}^{5}+A_{14} \hat{\omega}_{x}^{4}+A_{13} \hat{\omega}_{x}^{3}+A_{12} \hat{\omega}_{x}^{2}, \quad \hat{\omega}_{x} \leq T_{A 1} \\
A_{23} \hat{\omega}_{x}^{3}+A_{22} \hat{\omega}_{x}^{2}+A_{21} \hat{\omega}_{x}+A_{20}, \quad T_{A 1} \leq \hat{\omega}_{x} \leq T_{A 2} \\
A_{30}-A_{31} \exp \left(-A_{32} \hat{\omega}_{x}\right), \quad \hat{\omega}_{x} \geq T_{A 2}
\end{array}\right. \\
& B=\left\{1-\exp \left[-\left(35.6-30.4 \hat{\alpha}_{y}\right) \hat{\omega}_{y}\right]\right\}\left(1-0.71 \hat{\alpha}_{y}^{2}\right)\left\{\begin{array}{l}
B_{15} \hat{\omega}_{x}^{5}+B_{14} \hat{\omega}_{x}^{4}+B_{13} \hat{\omega}_{x}^{3}+B_{12} \hat{\omega}_{x}^{2}, \quad \hat{\omega}_{x} \leq T_{B 1} \\
B_{23} \hat{\omega}_{x}^{3}+B_{22} \hat{\omega}_{x}^{2}+B_{21} \hat{\omega}_{x}+B_{20}, \quad T_{B 1} \leq \hat{\omega}_{x} \leq T_{B 2} \\
B_{30}, \quad \hat{\omega}_{x} \geq T_{B 2}
\end{array}\right.
\end{aligned}
$$

The correlation coefficients in Eqs. (58) and (59) were determined by forcing continuity of the functions and their first derivatives at the transition points, while minimizing the RMS difference between the CFD solutions and the correlating functions. This produced the correlation coefficients given in Table 1. Using Eqs. (58) and (59) with the correlation coefficients given in Table 1 produces the results shown in Figs. 14 and 15. Note that Fig. 15 exhibits significantly more deviation between the CFD solutions and the correlation equation than does Fig. 14. This could be attributed to the fact that the relative uncertainty in the CFD solutions for the mean axial force is on the order of $3 \%$, whereas that for the mean required power is less than $0.5 \%$. These uncertainties were determined from results similar to those presented in Figs. 9 and 10.

Applying Eqs. (58) and (59) with the correlation coefficients given in Table 1 to Eqs. (48), (54), and (55) produces the results presented in Figs. 16-18, which are shown over the range of axial reduced frequencies that are typically encountered in the forward flight of birds. All results shown in Figs. 16-18 were obtained for a geometric angle-of-attack amplitude of $\hat{\omega}_{y}\left(1-\hat{\alpha}_{y}\right)=4.29^{\circ}$ and a mean lift coefficient of $\bar{C}_{L}=0.614$. Results from the Theodorsen model are included for comparison. The deviation between the CFD results and Eqs. (48), (54), and (55) for all cases in this study ranges from $-0.7 \%$ to $1.7 \%$ for the mean axial-force coefficient, from $-0.08 \%$ to $0.6 \%$ for the mean required-power coefficient, and from $-0.9 \%$ to $1.5 \%$ for the propulsive efficiency. Thus, in the range of reduced frequencies studied, the correlation matches the CFD results to within $2 \%$. Because the CFD uncertainty 
Table 1. Correlation coefficients for Eqs. (58) and (59).

\begin{tabular}{|lllllllll|}
\hline \hline$A_{15}=97.29135$ & $A_{23}=0.546031$ & $A_{30}=$ & 0.587284 & $B_{15}=-544.4944$ & $B_{23}=-14.76825$ & $B_{30}=-0.265167$ \\
$A_{14}=-43.97278$ & $A_{22}=-1.041703$ & $A_{31}=$ & 0.804087 & $B_{14}=49.2633$ & $B_{22}=11.25173$ & \\
$A_{13}=-17.06888$ & $A_{21}=$ & 0.961984 & $A_{32}=$ & 2.611038 & $B_{13}=109.3024$ & $B_{21}=-2.75989$ & \\
$A_{12}=$ & 9.97222 & $A_{20}=$ & 0.108206 & $T_{A 1}=$ & 0.332091 & $B_{12}=-26.2319$ & $B_{20}=-0.05111$ & $T_{B 1}=0.226322$ \\
& & & & & & $T_{B 2}=0.300902$ \\
\hline \hline
\end{tabular}

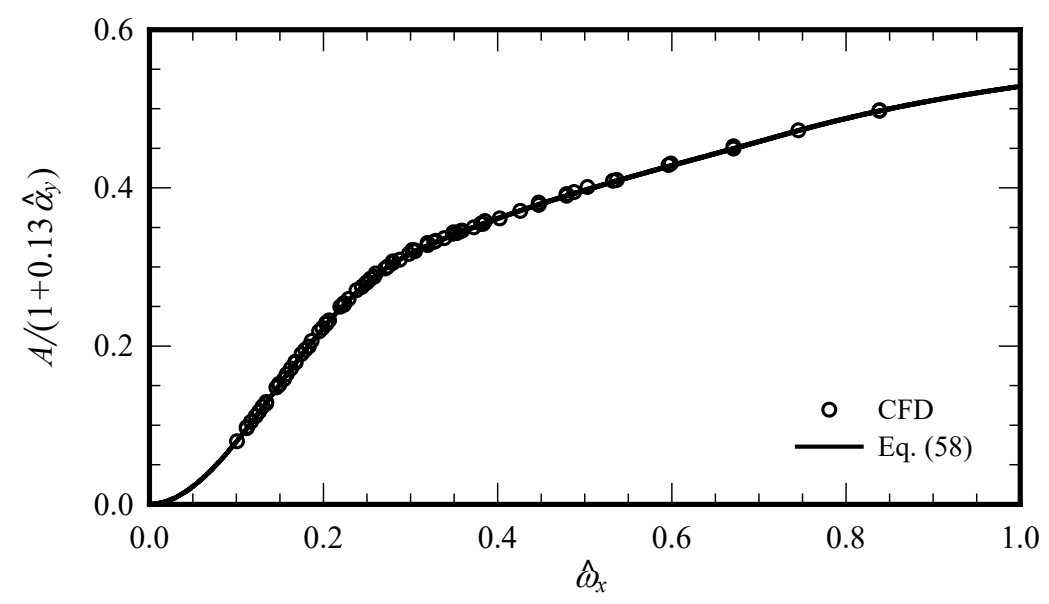

Figure 14. Comparison between the CFD solutions and the correlation with Eq. (58).

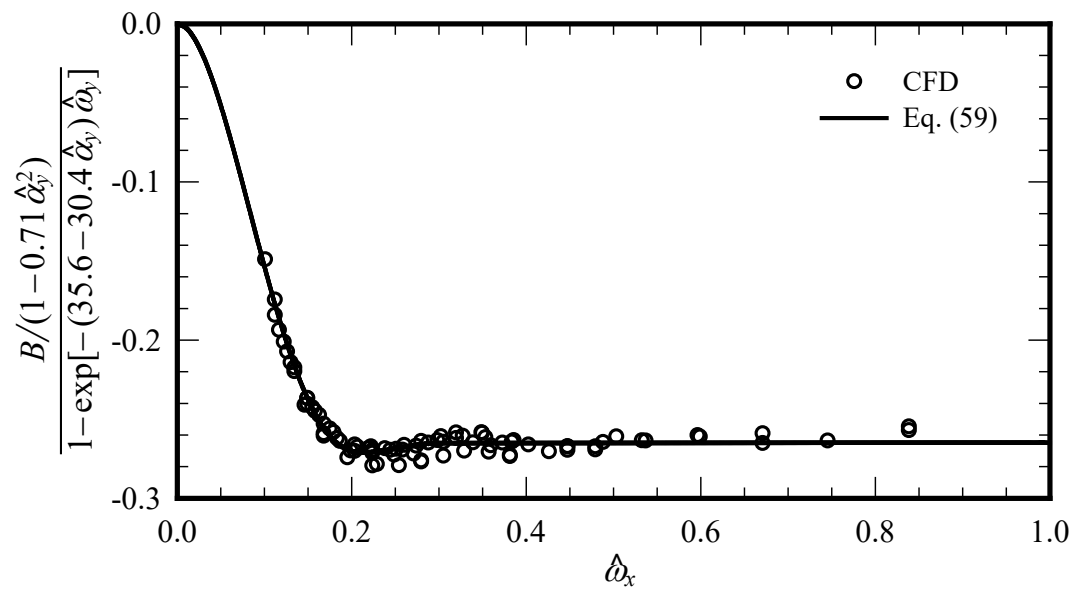

Figure 15. Comparison between the CFD solutions and the correlation with Eq. (59).

in the mean axial-force coefficient is on the order of $3 \%$ and that for the mean required-power coefficient is on the order of $1 \%$, we should expect the correlations presented here to provide a noticeable improvement over the Theodorsen model.

In summary, Eqs. (58) and (59) along with the coefficients given in Table 1 can be used in Eqs. (48), (54), and (55) to predict the mean axial-force coefficient, required-power coefficient, and propulsive efficiency of airfoils with sinusoidal pitching and plunging motion. Furthermore, the time-dependent induced angle of attack as well as the instantaneous force coefficients can be evaluated using Eqs. (40), (41), and (44). Because the model is written in terms of the airfoil aerodynamic properties $\widetilde{C}_{L, \alpha}$ and $\alpha_{L 0}$, this model can be used for any airfoil for which these properties are known, and is therefore not constrained to thin airfoils. Finally, this model can be implemented over a wide range of flapping frequencies. 


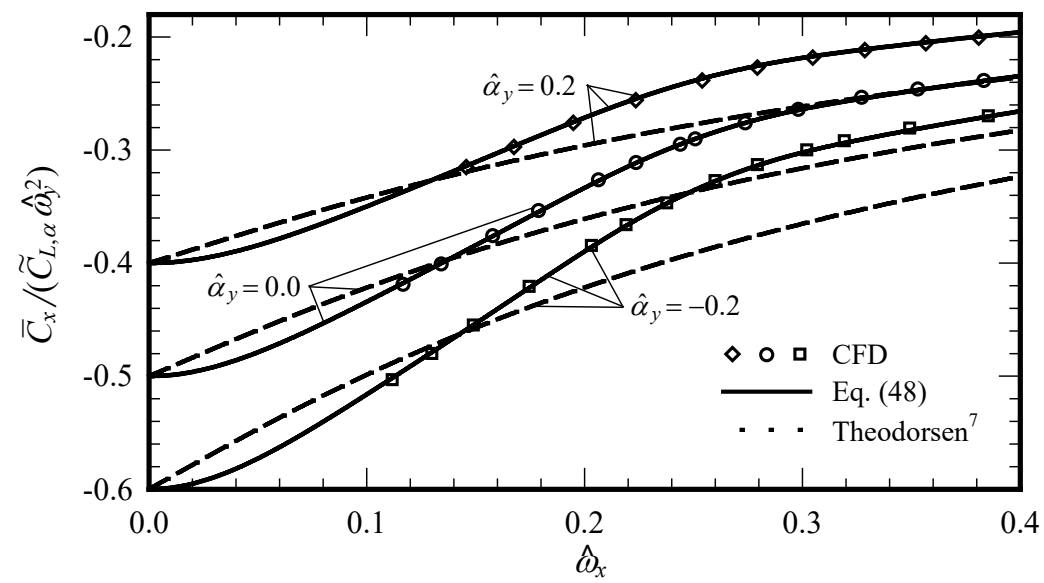

Figure 16. Comparison of the mean axial-force coefficient predicted from the CFD solutions, Eq. (48), and the model first presented by Theodorsen. ${ }^{7}$

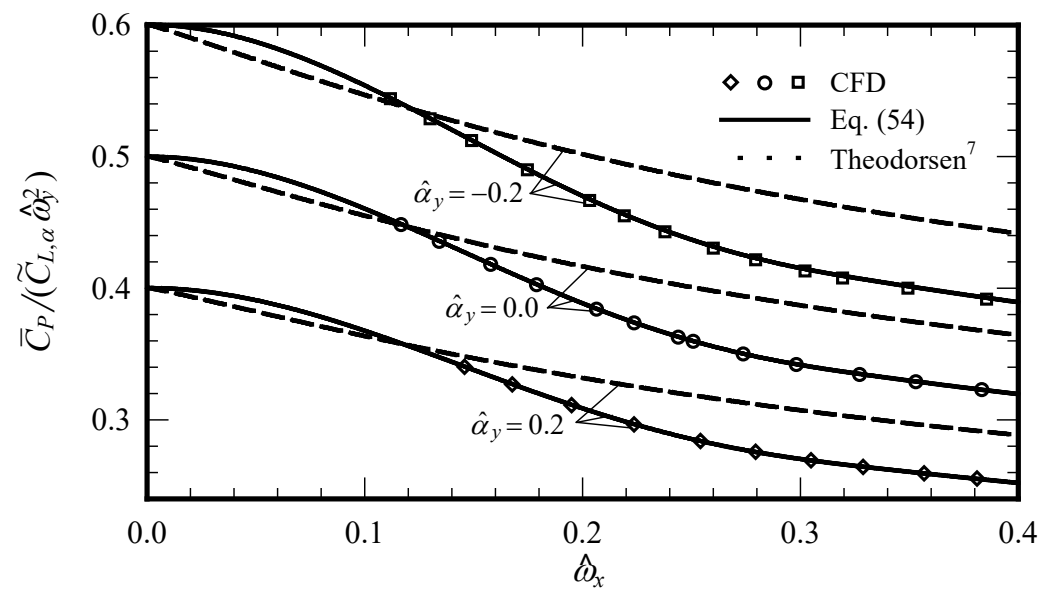

Figure 17. Comparison of the mean required-power coefficient predicted from the CFD solutions, Eq. (54), and the model first presented by Theodorsen. ${ }^{7}$

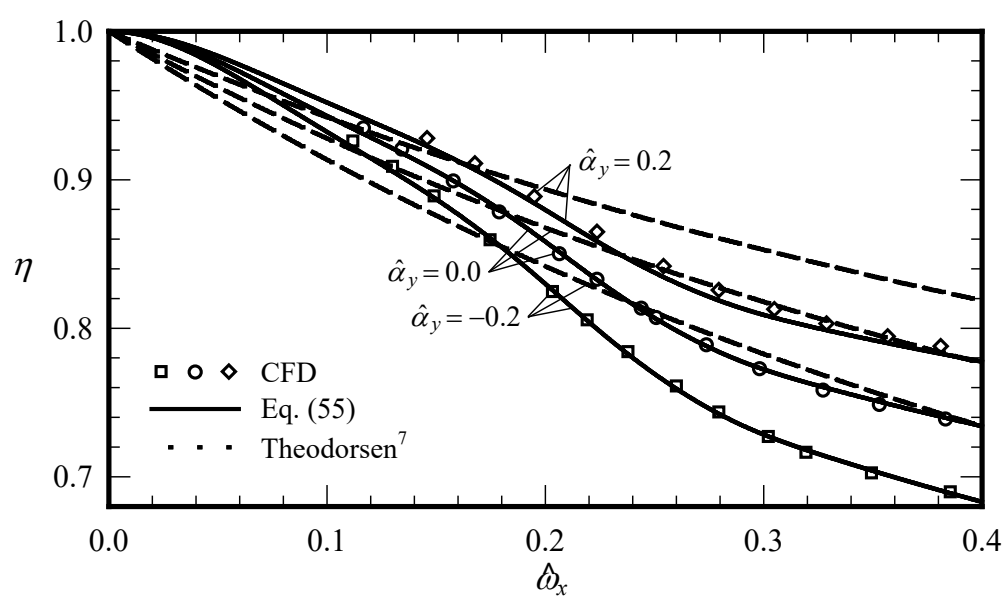

Figure 18. Comparison of the propulsive efficiency predicted from the CFD solutions, Eq. (55), and the model first presented by Theodorsen. ${ }^{7}$ 


\section{Conclusions}

A straight-forward summary of Theodorsen's ${ }^{7}$ flapping airfoil model has been presented using updated terminology and symbols. It has been shown that this model exhibits the correct asymptotic behavior at low flapping frequencies. However, in the high-frequency limit, Theodorsen's model predicts infinite amplitude oscillations in the aerodynamic forces and moments at finite geometric pitching and plunging angle-of-attack amplitudes. These high-frequency results are unrealistic.

Theodorsen's model has been compared to grid- and time-step-resolved inviscid CFD solutions in the range of flapping frequencies and amplitudes commonly encountered in the forward flight of birds. These results show that both axial and normal reduced frequencies are of significant importance. The axial reduced frequency is based on the chord length as defined in Eq. (52), and the normal reduced frequency is based on the aerodynamic-center plunging amplitude as defined in Eq. (20). The computational results agree reasonably well with the Theodorsen model at low flapping frequencies. In fact, for axial reduced frequencies in the range $\hat{\omega}_{x}<0.2$, Theodorsen's model agrees with all CFD results from this study to within $10 \%$. Even at higher frequencies, Theodorsen's model agrees with the numerical results to within $35 \%$.

An alternate approach to that taken by Theodorsen has also been presented. It has been shown here that the time-dependent aerodynamic forces acting on an airfoil undergoing sinusoidal flapping can be related to only two unknown Fourier coefficients. These Fourier coefficients were obtained in the present study from the grid- and time-step-resolved inviscid CFD solutions. Correlations for these Fourier coefficients were presented in Eqs. (58) and (59), which can be used with Eqs. (48), (54), and (55) to predict the mean axial-force coefficient, mean required-power coefficient, and mean propulsive efficiency for sinusoidal flapping of an airfoil with known geometry and known aerodynamic coefficients for steady flow. The algebraic relations that are given in Eqs. (58) and (59) were correlated with the CFD results to obtain the correlation coefficients given in Table 1. The Fourier coefficients were found to be strong functions of the axial reduced frequency, $\hat{\omega}_{x}$, but only weak functions of the normal reduced frequency, $\hat{\omega}_{y}$, and the geometric angle-of-attack ratio, $\hat{\alpha}_{y}$. Results obtained from the correlations match the CFD results to within $2.0 \%$ for the mean axial-force coefficient, mean required-power coefficient, and propulsive efficiency in the entire range of axial reduced frequencies studied and can be used as an improvement to the model presented by Theodorsen ${ }^{7}$ for predicting the propulsion of flapping airfoils.

\section{References}

${ }^{1}$ Knoller, R., "Die Gesetze des Luftwiderstandes," Flug- und Motortechnik (Wien), Vol. 3, No. 21, 1909, pp. 1-7.

${ }^{2}$ Betz, A., "Ein Beitrag zur Erklärung des Segelfluges," Zeitschrift für Flugtechnik und Motorluftschiffahrt, Vol. 3, 1912, pp. 269-272.

${ }^{3}$ Katzmayr, R., "Effect of Periodic Changes of Angle of Attack on Behavior of Airfoils," NACA TR-147, Oct. 1922.

${ }^{4}$ Wagner, H. "Über die Entstehung des dynamischen Auftriebs von Tragflügeln," Zeitschrift für Angewandte Mathematic und Mechanik, Vol. 5, No. 1, Feb. 1925, pp. 17-35.

${ }^{5}$ Glauert, H., "The Force and Moment on an Oscillating Aerofoil," Technical Report of the Aeronautical Research Committee, Vol. 2, R. \& M. No. 1242, 1931, pp. 742-758.

${ }^{6}$ von Kármán, T., and Burgers, J. M., "General Aerodynamic Theory — Perfect Fluids," Aerodynamic Theory, edited by Durand, W. F., Vol. 2, Springer, Berlin, 1935, pp. 293-310.

${ }^{7}$ Theodorsen, T., "General Theory of Aerodynamic Instability and the Mechanism of Flutter," NACA TR-496, Feb. 1935.

${ }^{8}$ Garrick, I. E., "Propulsion of a Flapping and Oscillating Airfoil," NACA TR-567, May 1936.

${ }^{9}$ Anderson, J. M., Streitlien, K., Barrett, D. S., and Triantafyllou M. S., "Oscillating Foils of High Propulsive Efficiency," Journal of Fluid Mechanics, Vol. 360, Apr. 1998, pp. 41-72.

${ }^{10}$ Lai, J. C., and Platzer, M. F., "Jet characteristics of a plunging airfoil,” AIAA Journal, Vol. 37, No. 12, 1999 , pp. $1529-1537$.

${ }^{11}$ Dickinson, M. H., Lehmann, F. O., and Sane, S. P., "Wing Rotation and the Aerodynamic Basis of Insect Flight," Science, Vol. 284, 1999, pp. 1954-1960.

${ }^{12}$ Von Ellenrieder, K. D., Parker, K, and Soria, J., "Flow Structures Behind a Heaving and Pitching Finite-Span Wing," Journal of Fluid Mechanics, Vol. 490, Sep. 2003, pp. 129-138.

${ }^{13}$ Tarascio, M. J., Ramasamy, M., Chopra, I., and Leishman, J. G., "Flow Visualization of Micro Air Vehicle Scaled InsectBased Flapping Wings," Journal of Aircraft, Vol. 42, No. 2, 2005, pp. 385-390.

${ }^{14}$ Ramasamy, M., and Leishman, J. G., "Phase-Locked Particle Image Velocimetry Measurements of a Flapping Wing," Journal of Aircraft, Vol. 43, No. 5, 2006, pp. 1867-1876.

${ }^{15}$ Heathcote, S. and Gursul, I., "Flexible Flapping Airfoil Propulsion at Low Reynolds Numbers," AIAA Journal, Vol. 45, No. 5, 2007, pp. 1066-1079.

${ }^{16}$ Hong, Y., and Altman, A., "Streamwise Vorticity in Simple Mechanical Flapping Wings," Journal of Aircraft, Vol. 44, No. 5, 2007, pp. 1588-1597. 
${ }^{17}$ Warkentin, J., and DeLaurier, J., "Experimental Aerodynamic Study of Tandem Flapping Membrane Wings," Journal of Aircraft, Vol. 44, No. 5, 2007, pp. 1653-1661.

${ }^{18}$ Hong, Y., and Altman, A., "Lift from Spanwise Flow in Simple Flapping Wings," Journal of Aircraft, Vol. 45, No. 3, 2008, pp. 1206-1216.

${ }^{19}$ Yang, L. J., Hsu, C. K., Han, H. C., and Miao, J. M., "Light Flapping Micro Aerial Vehicle Using Electrical-Discharge WireCutting Technique," Journal of Aircraft, Vol. 46, No. 5, 2009, pp. 1866-1874.

${ }^{20}$ Grauer, J., Ulrich, E., Hubbard, J., Pines, D., and Humbert J. S., "Testing and System Identification of an Ornithopter in Longitudinal Flight," Journal of Aircraft, Vol. 48, No. 2, 2011, pp. 660-667.

${ }^{21}$ Yang, L. J., Ko, A. F., and Hsu, C. K., "Wing Stiffness on Light Flapping Micro Aerial Vehicles," Journal of Aircraft, Vol. 49, No. 2, 2012, pp. 423-431.

${ }^{22}$ Peng, D., and Milano, M., "Lift Generation with Optimal Elastic Pitching for a Flapping Plate," Journal of Fluid Mechanics, Vol. 717, Feb. 2013, pp. R1-1-R1-13.

${ }^{23}$ Pitt Ford, C. W., and Babinsky, H., "Lift and the Leading-Edge Vortex," Journal of Fluid Mechanics, Vol. 720, Apr. 2013, pp. 280-313.

${ }^{24}$ Izraelevitz, J. S., and Triantafyllou, M. S., “Adding In-Line Motion and Model-Based Optimization Offers Exceptional Force Control Authority in Flapping Foils," Journal of Fluid Mechanics, Vol. 742, Mar. 2014, pp. 5-34.

${ }^{25}$ Lan, C. E., "The Unsteady Quasi-Vortex-Lattice Method with Applications to Animal Propulsion," Journal of Fluid Mechanics, Vol. 93, Aug. 1979, pp. 747-765.

${ }^{26}$ Smith, M., "Simulating Moth Wing Aerodynamics: Towards the Development of Flapping-Wing Technology," AIAA Journal, Vol. 34, No. 7, 1996, pp. 1348-1355.

${ }^{27}$ Vest, M., and Katz, J., "Unsteady Aerodynamic Model of Flapping Wings," AIAA Journal, Vol. 34, No. 7, 1996, pp. 14351440 .

${ }^{28}$ Hall, K., Pigott, S., and Hall, S., "Power Requirements for Large-Amplitude Flapping Flight," Journal of Aircraft, Vol. 35, No. 3, 1998, pp. 352-361.

${ }^{29}$ Fritz, T. E., and Long, L. N., "Object-Oriented Unsteady Vortex Lattice Method for Flapping Flight," Journal of Aircraft, Vol. 41, No. 6, 2004, pp. 1275-1290.

${ }^{30}$ Isogai, K., and Harino, Y., “Optimum Aeroelastic Design of a Flapping Wing,” Journal of Aircraft, Vol. 44, No. 6, 2007 , pp. 2040-2048.

${ }^{31}$ Ansari, S. A., Knowles, K., and Zbikowski, R., "Insectlike Flapping Wings in the Hover Part I: Effect of Wing Kinematics," Journal of Aircraft, Vol. 45, No. 4, 2008, pp. 1945-1954.

${ }^{32}$ Ansari, S. A., Knowles, K., and Zbikowski, R., "Insectlike Flapping Wings in the Hover Part II: Effect of Wing Geometry," Journal of Aircraft, Vol. 45, No. 5, 2008, pp. 1976-1990.

${ }^{33}$ Gulcat, U., "Propulsive Force of a Flexible Flapping Thin Airfoil," Journal of Aircraft, Vol. 46, No. 2, 2009, pp. $465-473$.

${ }^{34}$ Stanford, B. K., and Beran, P. S., , "Analytical Sensitivity Analysis of an Unsteady Vortex-Lattice Method for FlappingWing Optimization," Journal of Aircraft, Vol. 47, No. 2, 2010, pp. 647-662.

${ }^{35}$ Stanford, B., Kurdi, M., Beran, P., and McClung, A., "Shape, Structure, and Kinematic Parameterization of a Power-Optimal Hovering Wing," Journal of Aircraft, Vol. 49, No. 6, 2012, pp. 1687-1699.

${ }^{36}$ Quinn, D. B., Moored, K. W., Dewey, P. A., and Smits, A. J., “Unsteady Propulsion Near a Solid Boundary,” Journal of Fluid Mechanics, Vol. 742, Mar. 2014, pp. 152-170.

${ }^{37}$ Ramesh, K., Gopalarathnam, A., Granlund, K., Ol, M. V., and Edwards, J. R., "Discrete-Vortex Method with Novel Shedding Criterion for Unsteady Aerofoil Flows with Intermittent Leading-Edge Vortex Shedding," Journal of Fluid Mechanics, Vol. 751, July 2014, pp. 500-538.

${ }^{38}$ Tuncer, I. H., and Platzer, M. F., “Computational Study of Flapping Airfoil Aerodynamics," Journal of Aircraft, Vol. 37 , No. 2, 2000, pp. 514-520.

${ }^{39}$ Lewin, G. C., and Haj-Hariri, H., "Modelling Thrust Generation of a Two-Dimensional Heaving Airfoil in a Viscous Flow," Journal of Fluid Mechanics, Vol. 492, Oct. 2003, pp. 339-362

${ }^{40}$ Tuncer, I. H., and Kaya, M., "Thrust Generation Caused by Flapping Airfoils in a Biplane Configuration," Journal of Aircraft, Vol. 40, No. 2, 2003, pp. 509-515.

${ }^{41}$ Young, J., and Lai, J., "Oscillation Frequency and Amplitude Effects on the Wake of a Plunging Airfoil," AIAA Journal, Vol. 42, No. 10, 2004, pp. 2042-2052.

${ }^{42}$ Blondeaux, P., Fornarelli, F., Guglielmini, L., Triantafyllou, M. S., and Verzicco, R., "Numerical Experiments on Flapping Foils Mimicking Fish-like Locomotion,” Physics of Fluids, Vol. 17, 2005, pp. 113601-1-113601-12.

${ }^{43}$ Tuncer, I., and Kaya, M., "Optimization of Flapping Airfoils for Maximum Thrust and Propulsive Efficiency," AIAA Journal, Vol. 43, No. 11, 2005, pp. 2329-2336.

${ }^{44}$ Young, J. and Lai, J. C. S., "Mechanisms Influencing the Efficiency of Oscillating Airfoil Propulsion," AIAA Journal, Vol. 45, No. 7, 2007, pp. 1695-1702.

${ }^{45}$ Cizmas, P., and Gargoloff, J., "Mesh Generation and Deformation Algorithm for Aeroelasticity Simulations," Journal of Aircraft, Vol. 45, No. 3, 2008, pp. 1062-1066. 
${ }^{46}$ Lee, J., Kim, J., and Kim, C., "Numerical Study on the Unsteady-Force-Generation Mechanism of Insect Flapping Motion," AIAA Journal, Vol. 46, No. 7, 2008, pp. 1835-1848.

${ }^{47}$ Brunton, S. L. and Rowley, C. W., "Modeling the Unsteady Aerodynamic Forces on Small-Scale Wings," 47 th AIAA Aerospace Sciences Meeting, 5-8 January 2009, Orlando, Florida, AIAA-2009-1127.

${ }^{48}$ Kaya, M., Tuncer, I. H., Jones, K. D., and Platzer, M. F., "Optimization of Flapping Motion Parameters for Two Airfoils in a Biplane Configuration,” Journal of Aircraft, Vol. 46, No. 2, 2009, pp. 583-592.

${ }^{49}$ Miao, J. M., Sun, W. H., and Tai, C. H., "Numerical Analysis on Aerodynamic Force Generation of Biplane CounterFlapping Flexible Airfoils," Journal of Aircraft, Vol. 46, No. 5, 2009, pp. 1785-1794.

${ }^{50}$ Roget, B., Sitaraman, J., Harmon, R., Grauer, J., Hubbard, J., and Humbert, S., "Computational Study of Flexible Wing Ornithopter Flight," Journal of Aircraft, Vol. 46, No. 6, 2009, pp. 2016-2031.

${ }^{51}$ Chandar, D. D. J., and Damodaran, M., "Computation of Unsteady Low Reynolds Number Free-Flight Aerodynamics of Flapping Wings," Journal of Aircraft, Vol. 47, No. 1, 2010, pp. 141-150.

${ }^{52} \mathrm{Li}$, G., and Lu, X., "Force and Power of Flapping Plates in a Fluid," Journal of Fluid Mechanics, Vol. 712, Dec. 2012, pp. 598-613.

${ }^{53}$ Zheng, L., Hedrick, T. L., and Mittal, R., “A Multi-Fidelity Modelling Approach for Evaluation and Optimization of Wing Stroke Aerodynamics in Flapping Flight,” Journal of Fluid Mechanics, Vol. 721, Apr. 2013, pp. 118-154.

${ }^{54}$ Harbig, R. R., Sheridan, J., and Thompson, M. C., "The Role of Advance Ratio and Aspect Ratio in Determining LeadingEdge Vortex Stability for Flapping Flight," Journal of Fluid Mechanics, Vol. 751, July 2014, pp. 71-105.

${ }^{55}$ Baik, Y. S., Bernal, L. P., Granlund, K., and Ol, M. V., "Unsteady Force Generation and Vortex Dynamics of Pitching and Plunging Aerofoils," Journal of Fluid Mechanics, Vol. 709, Oct. 2012, pp. 37-68.

${ }^{56}$ Brunton, S. L., Rowley, C. W., and Williams, D. R., "Reduced-Order Unsteady Aerodynamic Models at Low Reynolds Numbers," Journal of Fluid Mechanics, Vol. 724, June 2013, pp. 203-233.

${ }^{57}$ CD-adapco, "User Guide: Star-CCM+ Version 8.06," 2013, pp. 1-13268.

${ }^{58}$ Phillips, W. F., Fugal, S. R., and Spall, R. E., "Minimizing Induced Drag with Wing Twist, Computational-Fluid-Dynamics Validation," Journal of Aircraft, Vol. 43, No. 2, 2006, pp. 437-444. 